\title{
Evaluation of interfacial excess contributions in different phase-field models for elastically inhomogeneous systems
}

\author{
A Durga, P Wollants and N Moelans \\ Department of Metallurgy and Materials Engineering, Faculty of Engineering, \\ KU Leuven, Kasteelpark Arenberg 44 Bus 2450, 3001 Heverlee, Belgium \\ E-mail: durga.ananthanarayanan@mtm.kuleuven.be
}

\begin{abstract}
In elastically inhomogeneous solid materials, the presence of strains causes changes in both morphology and phase equilibria, thereby changing the mechanical and chemical properties. For any given inital phase- and grain-structure, it is difficult to determine experimentally or analytically these changes in properties. Phase-field models coupled with microelasticity theory can be used to predict the morphological and chemical evolution of such strained systems, but their accuracy with respect to interfacial excess contributions has not been tested extensively. In this study, we analyse three existing phase-field schemes for coherent two-phase model systems and a $\mathrm{Cu}_{6} \mathrm{Sn}_{5}-\mathrm{Bct}-\mathrm{Sn}$ system. We compare the chemical composition and stress state obtained in the simulations with analytical values calculated from Johnson's [Johnson W C 1987 Metall. Trans. A 18A 233-47] model. All schemes reproduce the shift in chemical composition, but not the strains. This deviation is due to excess interfacial energy, stresses, and strains not present in the analytical results, since all three schemes are based on assumptions different from the stress and strain relations at equilibrium. Based on this analysis, we introduce a new scheme which is consistent with the analytical calculations. We validate for the model system that this new scheme quantitatively predicts the morphological and chemical evolution, without any interfacial excess contributions and independent of the diffuse interface width.
\end{abstract}

PACS numbers: 64.70.kd, 61.72.-y, 46.25.-y, 68.35.-p

Submitted to: Modelling Simulation Mater. Sci. Eng.

\section{Introduction}

In the field of materials science, analysis of microstructural evolution in solid-state systems is an important problem, as it has applications in different fields like ferrous metallurgy, superalloys and solders. A significant feature of studying phenomena at the solid-state, compared to fluids, is the presence of elastic strains and stresses in the material, which can greatly alter the chemical behaviour, morphology, growth kinetics and mechanical behaviour of the system. 
In the presence of elastic strains, the chemical equilibrium of the system shifts [1] due to a shift in the Gibbs energy curves. In previous studies, Cahn and Larché [1] have developed a method to calculate the phase diagram for a binary two-phase coherent system with equal stiffnesses and Pfeifer and Voorhees [2] for inhomogeneous binary two-phase systems. Johnson [3] has further developed a model to compute the change in equilibrium composition for an inhomogeneous system with a second-phase precipitate in an infinite supersaturated matrix in the presence of external and internal strains. However, in general, the change in chemical composition and the elastic strain generated due to misfit cannot be calculated analytically for complex, finite, realistic systems.

Phase-field modelling combined with micro-elasticity theory can be used as a tool to study the elastic effects on solid-state phase transformations and grain growth for any arbitrary morphology [4-6]. The elastic energy density is formulated in the smallstrain limit as $f^{e l}=\frac{1}{2} \epsilon_{i j}^{e l} C_{i j k l} \epsilon_{k l}^{e l}$, where $C_{i j k l}$ is the stiffness, a fourth-order tensor, and the elastic strain $\epsilon_{k l}^{e l}$, a second-order tensor, is the difference between total strain $\epsilon_{k l}^{t o t}$ and eigenstrain $\epsilon_{k l}^{*}$ (Einstein summation notation is used for all tensorial relations including the above). The elastic stresses and strains have been defined in the diffuse interface by interpolating their values in the bulk phases using a number of schemes, three of which we discuss below. The bulk elastic and chemical properties predicted by these phase-field simulations are influenced by interfacial excess contributions, if any. Even though phase-field models coupled with micro-elasticity theory have been applied to many realistic systems, it still remains a challenge to formulate the elastic energy in such a way that it creates no excess interfacial energy [11]. This excess interfacial energy scales with the diffuse interface width and therefore, the phase-field simulations are no longer quantitative. Analysing this effect, which has not yet been studied extensively, and finding an interpolation scheme that avoids this is a main concern of this article.

The most widely applied interpolation scheme is Khachaturyan's (KHS) [7], wherein the stiffness $C_{i j k l}$ and eigenstrain $\epsilon_{k l}^{*}$ are interpolated as $C_{i j k l}=\sum_{\alpha} \phi_{\alpha} C_{i j k l}^{\alpha}$ and $\epsilon_{k l}^{*}=\sum_{\alpha} \phi_{\alpha} \epsilon_{k l}^{*, \alpha}$ respectively, where $\phi_{i}(i=\alpha, \beta, \ldots)$ are the interpolation functions and $\alpha, \beta, \ldots$ the different phases. This scheme has been implemented in phase-field models to study a wide variety of problems such as spinodal decomposition in elastically inhomogeneous thin films [8], growth of solid-state dendrites [9] and grain boundary migration in $\mathrm{Cu}$ bicrystals [10].

Another scheme is Steinbach-Apel's (SAS) [11], wherein the elastic strains are interpolated as $\epsilon_{k l}^{e l}=\sum_{\alpha} \phi_{\alpha} \epsilon_{k l}^{e l, \alpha}$. For the interpolation of stiffnesses, elastic stress is assumed to be equal in all the phases (Reuss-Sachs' condition) at every point in the interface. This gives rise to an interpolation of the compliances, the inverse of the stiffnesses, as $S_{i j k l}=\sum_{\alpha} \phi_{\alpha} S_{i j k l}^{\alpha}$. This scheme has been used to study different problems like pearlite formation in $\mathrm{Fe}-\mathrm{C}$ [12], growth of $\mathrm{Ni}_{4} \mathrm{Ti}_{3}$ particles in $\mathrm{Ni}-\mathrm{Ti}$ shape memory alloys [13] and grain growth and texture evolution in $\mathrm{Cu}$ [14]. 
Ammar et al. [15] have implemented KHS and two other alternative schemes, one based on Voigt-Taylor's assumptions [16] and the other on Reuss-Sachs' assumptions, but different from that proposed by Steinbach and Apel [11]. In the Voigt-Taylor's scheme (VTS) $[15,16]$, the total strain is assumed to be equal in all the phases at every point in the interface. Furthermore, the elastic stress $\sigma_{k l}$ is interpolated as

$\sigma_{k l}=\sum_{\alpha} \phi_{\alpha} \sigma_{k l}^{\alpha}$. They found that, for elastically homogeneous systems, the VTS and KHS show the correct change in chemical composition, whereas their Reuss-Sachs' scheme does not.

KHS and SAS were implemented in previous simulations by some of the authors [17] for $\mathrm{Cu}_{6} \mathrm{Sn}_{5}$ precipitate evolution in Bct-Sn matrix of a $\mathrm{Cu}-\mathrm{Sn}$ binary alloy. The stresses, strains and morphology predicted by the two schemes were different, whereas the compositions predicted were equal.

In this paper, a 2-D binary two-phase inhomogeneous system with a specific geometry is considered, for which the elastic strains and the change in equilibrium composition are calculated analytically using Johnson's [3] approach. KHS, SAS and a slightly modified VTS are implemented and analysed for this system. The $\mathrm{Cu}_{6} \mathrm{Sn}_{5^{-}}$ Bct-Sn system is further studied in this paper using KHS, SAS and VTS. The main aim of our work is to find an interpolation scheme that gives results consistent with the analytical model independent of the value of the diffuse interface width, so that it can be applied to more complex, realistic morphologies for which analytical calculations are not possible.

The rest of the paper is organised as follows. In section 2, the heterogeneous strain generated due to misfit and change in composition due to elastic strains are calculated analytically for the model 2-D system. In section 3, the formulation of the phase-field model for the three schemes, along with the method used to solve the elasticity equations, is described. Simulation results obtained for a realistic $\mathrm{Cu}_{6} \mathrm{Sn}_{5}-\mathrm{Bct}$-Sn alloy system and several model systems for which the stiffnesses and eigenstrains are of different forms are presented in section 4. Bulk properties and interfacial excess quantities are computed and compared with the analytical results. In section 5, an alternative scheme with interfacial conditions in agreement with those in the analytical model is proposed and validated for the model $2-\mathrm{D}$ system, followed by the main conclusions from this work in section 6 .

\section{Analytical sharp interface models}

Johnson [3] gave a sharp interface description to calculate the mechanical and chemical equilibrium analytically for an elastically inhomogeneous solid-state system with a second-phase spherical precipitate in an infinite supersaturated matrix. We apply a similar procedure here, considering a $2-\mathrm{D}$ binary two-phase $(\alpha-\beta)$ rectangular system with equal areas of the two phases $\left(A_{\alpha}=A_{\beta}\right)$, as shown in figure $1(\mathrm{a})$.

The interface between the two phases is assumed to be coherent. Undeformed $\alpha$ 
phase is chosen as the reference state $\left(\epsilon_{k l}^{*, \alpha}=0\right)$ and non-zero eigenstrains are present in the $\beta$ phase only $\left(\epsilon_{k l}^{*, \beta} \neq 0\right)$. The elastic moduli for the two phases are chosen such that the components $C_{1112}$ and $C_{2212}$ are zero, which is valid for most crystal structures. The applied and eigenstrains are assumed to have zero shear components.

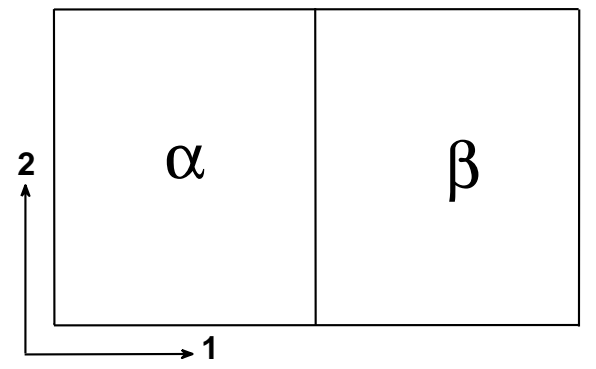

(a)

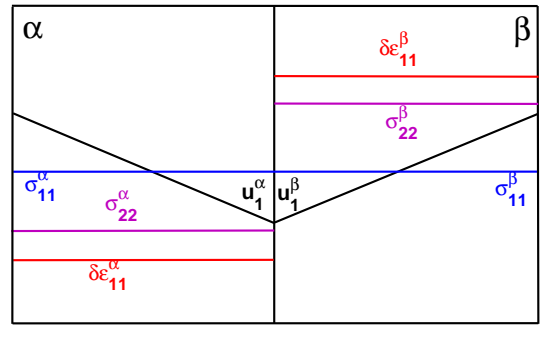

(b)

Figure 1. Illustration of the binary 2-phase 2-D system considered in this study: (a) Configuration. (b) Strain and stress profiles as calculated from Johnson's model (only non-zero quantities displayed).

\subsection{Mechanical equilibrium}

In the small strain limit, elastic stress is given by Hooke's law:

$$
\sigma_{i j}=C_{i j k l} \epsilon_{k l}^{e l}
$$

The elastic strain for each phase is defined as the difference between the total strain, and the eigenstrain or stress-free strain $\epsilon_{k l}^{*}$ :

$$
\epsilon_{k l}^{e l}=\epsilon_{k l}^{t o t}-\epsilon_{k l}^{*}
$$

Here, we consider only eigenstrain arising from the difference in lattice parameters of the two phases. Moreover, following Khachaturyan's approach [7], the total strain is considered as the sum of the homogeneous or applied strain $\overline{\epsilon_{k l}}$, and local heterogeneous strain $\delta \epsilon_{k l}$ :

$$
\epsilon_{k l}^{t o t}=\overline{\epsilon_{k l}}+\delta \epsilon_{k l}
$$

with the homogeneous strain defined such that

$$
\int_{V} \delta \epsilon_{i j} \mathrm{~d}^{3} r=0
$$

and the heterogeneous strain related to the local displacement vectors as:

$$
\delta \epsilon_{k l}=\frac{1}{2}\left[\frac{\partial u_{k}(\vec{r})}{\partial r_{l}}+\frac{\partial u_{l}(\vec{r})}{\partial r_{k}}\right]
$$

where $u_{i}(\vec{r})$ is the $i$ th component of the displacement vector. 
When both phases are in equilibrium, the following conditions should be satisfied [3]:

$$
\begin{aligned}
& u_{i}^{\alpha}=u_{i}^{\beta} \text { (continuity at the interface }+ \text { no slip) } \\
& \frac{\partial \sigma_{i j}}{\partial r_{j}}=0 \text { (mechanical equilibrium everywhere) } \\
& \sigma_{i j}^{\alpha} n_{j}^{\alpha}+\sigma_{i j}^{\beta} n_{j}^{\beta}=0 \text { (continuity of tractions at the interface) }
\end{aligned}
$$

where $n_{j}^{\alpha}$ and $n_{j}^{\beta}$ are the outward unit normals from $\alpha$ and $\beta$ respectively at the interface. Because of the system geometry and with zero shear components of homogeneous and eigenstrains, $\epsilon_{12}^{e l, \alpha}=0$. Since $C_{1112}=C_{2212}=0$, it follows from equation (1) that $\sigma_{12}^{\alpha}=$ 0 . Moreover, from the no-slip condition (6), $u_{2}^{\alpha}=u_{2}^{\beta}=0$. Therefore, $\delta \epsilon_{22}^{\alpha}=\delta \epsilon_{22}^{\beta}=0$. Equation (7) for $\mathrm{i}=1$ becomes $\partial \sigma_{11}^{\alpha} / \partial r_{1}=0$ or $\partial\left(C_{1111}^{\alpha} \delta \epsilon_{11}^{\alpha}+C_{1122}^{\alpha} \delta \epsilon_{22}^{\alpha}+C_{1112}^{\alpha} \delta \epsilon_{12}^{\alpha}\right) / \partial r_{1}$ $=0$. From (5), $\partial^{2} u_{1}^{\alpha} / \partial r_{1}^{2}=0$. Therefore, $\delta \epsilon_{11}^{\alpha}$ is a constant and $u_{1}$ varies linearly with position in the $\alpha$ phase. Similarly, $\delta \epsilon_{11}^{\beta}$ is a constant and $u_{1}^{\beta}$ varies linearly with position in the $\beta$ phase.

Evaluation of equation (4) for $i=j=1$ then gives

$$
\begin{aligned}
& \int_{A_{\alpha}} \delta \epsilon_{11}^{\alpha} d^{3} r+\int_{A_{\beta}} \delta \epsilon_{11}^{\beta} d^{3} r=0 \\
& \delta \epsilon_{11}^{\alpha}=-\delta \epsilon_{11}^{\beta}
\end{aligned}
$$

since the areas of $\alpha$ and $\beta$ are equal. Furthermore, since $n_{1}^{\alpha}=-n_{1}^{\beta}$, equation (8) gives $\sigma_{11}^{\alpha}=\sigma_{11}^{\beta}$. Therefore, using (1)-(3), we get

$$
\delta \epsilon_{11}^{\alpha}=\frac{C_{1111}^{\beta}\left(\overline{\epsilon_{11}}-\epsilon_{11}^{*, \beta}\right)+C_{1122}^{\beta}\left(\overline{\epsilon_{22}}-\epsilon_{22}^{*, \beta}\right)-C_{1111}^{\alpha} \overline{\epsilon_{11}}-C_{1122}^{\alpha} \overline{\epsilon_{22}}}{C_{1111}^{\alpha}+C_{1111}^{\beta}\left(A_{\alpha} / A_{\beta}\right)}
$$

Displacements, strain and stress profiles calculated using this sharp interface description are illustrated in Figure 1(b). Only the quantities that are non-zero are shown.

\subsection{Chemical equilibrium}

In the presence of elastic stresses, the new equilibrium chemical compositions, $c_{\alpha}^{e}$ and $c_{\beta}^{e}$, can be obtained using the derivation of Johnson [3] assuming infinite interface curvature:

$$
\begin{aligned}
& c_{\alpha}^{e}=c_{\alpha, 0}+\frac{\left(\sigma_{k l}^{\beta} \epsilon_{k l}^{e l, \beta}-\sigma_{k l}^{\alpha} \epsilon_{k l, \alpha}^{e l, \alpha}\right) / 2+\left(\epsilon_{k l}^{e l, \alpha}-\epsilon_{k l}^{t o t, \beta}\right) \sigma_{k l}^{\alpha}}{A^{\alpha}\left(c_{\beta, 0}-c_{\alpha, 0}\right)} \\
& c_{\beta}^{e}=c_{\beta, 0}+\frac{\left(\sigma_{k l}^{\beta} \epsilon_{k l}^{e l, \beta}-\sigma_{k l}^{\alpha} \epsilon_{k l}^{e l, \alpha}\right) / 2+\left(\epsilon_{k l}^{e l, \alpha}-\epsilon_{k l}^{t o t, \beta}\right) \sigma_{k l}^{\alpha}}{A^{\beta}\left(c_{\beta, 0}-c_{\alpha, 0}\right)}
\end{aligned}
$$

Here, $A^{\alpha}$ and $A^{\beta}$ are the second derivatives of the chemical energy densities $f_{\alpha}^{c h}$ and $f_{\beta}^{c h}$ of the two phases respectively and $c_{\alpha, 0}$ and $c_{\beta, 0}$ are the equilibrium compositions of the two phases in the absence of strains. 


\section{Phase-field model description}

We take up a binary (A-B) two-phase $(\alpha-\beta)$ solid-state coherent system, in the same configuration as figure 1(a), for performing phase-field simulations. The driving forces from chemical and elastic strain energies are considered. The system can be described by one independent composition variable, $c$, taken as the molar fraction of element $\mathrm{B}$, and one phase-field variable $\phi$, which takes the value 1 in the bulk of $\alpha$ phase, 0 in the bulk of $\beta$ phase and varies smoothly across the interface between the two phases. The total free energy functional for the system is formulated as:

$$
F=\int_{V}\left(f^{i n t}+f^{c h}+f^{e l}\right) \mathrm{d} V
$$

where $f^{i n t}$ is the interfacial energy density, $f^{c h}$, the chemical free energy density, and $f^{e l}$, the elastic energy density. The interfacial and chemical energies are formulated as per the Kim et al. [18] model and three different schemes are considered for the elastic energy formulation as described below.

\subsection{Interfacial energy density}

The interfacial energy density is of the form [18]:

$$
\begin{aligned}
& f^{i n t}=\frac{\kappa}{2}(\vec{\nabla} \phi)^{2}+W g(\phi), \\
& \text { giving } \frac{\partial f^{i n t}}{\partial \phi}=-\kappa \vec{\nabla}^{2} \phi+W g^{\prime}(\phi)
\end{aligned}
$$

where $\kappa$ is the gradient energy coefficient, $g(\phi)=\phi^{2}(1-\phi)^{2}$, the double-well function, and $\mathrm{W}$ the depth of the double-well. $\kappa$ and $\mathrm{W}$ are parameters related to the interfacial energy $\sigma_{\text {int }}$ and the interfacial width $l_{\text {int }}$ as $\sigma_{\text {int }}=\frac{1}{3} \sqrt{\kappa W / 2}$ and $l_{\text {int }}=\alpha \sqrt{2 \kappa / W}$ respectively. With $\alpha=2.2$, the interfacial width is defined as the length across which $\phi$ changes from 0.1 to 0.9 when the system is at equilibrium.

\subsection{Chemical energy density}

The chemical energy density is interpolated between the energy densities of the two phases [18] using a function $p(\phi)=\phi^{3}\left(6 \phi^{2}-15 \phi+10\right)[19]$ :

$$
f^{c h}=p(\phi) f_{\alpha}^{c h}+[1-p(\phi)] f_{\beta}^{c h}
$$

In this study, $f_{\alpha}^{c h}$ and $f_{\beta}^{c h}$ are taken as parabolic functions of the composition:

$$
f_{\alpha}^{c h}=\frac{A^{\alpha}}{2}\left(c_{\alpha}-c_{\alpha, 0}\right)^{2} ; f_{\beta}^{c h}=\frac{A^{\beta}}{2}\left(c_{\beta}-c_{\beta, 0}\right)^{2}
$$

where $A^{\alpha}, A^{\beta}, c_{\alpha, 0}$, and $c_{\beta, 0}$ are constants. Phase compositions $c_{\alpha}$ and $c_{\beta}$ are introduced, which relate the overall composition $c$ to the phase-field variable $\phi$ as:

$$
c=p(\phi) c_{\alpha}+[1-p(\phi)] c_{\beta}
$$


It is postulated by Kim et al. [18] that, at every point in the system, there is equal diffusion potential, i.e.,

$$
\mu_{\alpha}=\mu_{\beta}=\mu=\frac{\partial f^{c h}}{\partial c}=A^{\alpha}\left(c_{\alpha}-c_{\alpha, 0}\right)=A^{\beta}\left(c_{\beta}-c_{\beta, 0}\right)
$$

The derivative of the chemical energy density with respect to $\phi$ is given by (see Appendix A):

$$
\frac{\partial f^{c h}}{\partial \phi}=p^{\prime}(\phi)\left[f_{\beta}^{c h}-f_{\alpha}^{c h}+\mu_{\alpha}\left(c_{\beta, 0}-c_{\alpha, 0}\right)\right]
$$

\subsection{Elastic energy density}

Elastic energy can be incorporated in the framework of phase-field modelling using an appropriate interpolation scheme for defining the elastic strain, stress, stiffness or energy at the interface using their values in the bulk of the two phases. In this work, Khachaturyan's scheme (KHS), Steinbach-Apel's scheme (SAS) and a Voigt-Taylor's scheme (VTS) are considered for analysis. All elastic properties are assumed to be functions of the phase-field $\phi$ and independent of the composition.

3.3.1. Khachaturyan's scheme (KHS) In KHS [7,15], the stiffness is interpolated using the interpolation function $p(\phi)$ as:

$$
C_{i j k l}=p(\phi) C_{i j k l}^{\alpha}+[1-p(\phi)] C_{i j k l}^{\beta}
$$

where $C_{i j k l}^{\alpha}$ and $C_{i j k l}^{\beta}$ are constants. The eigenstrain is also interpolated as $\epsilon_{k l}^{*}=$ $p(\phi) \epsilon_{k l}^{*, \alpha}+[1-p(\phi)] \epsilon_{k l}^{*, \beta}$. The elastic energy density and its derivative with respect to $\phi$ are given by:

$$
\begin{aligned}
& f^{e l}=\frac{1}{2} \epsilon_{i j}^{e l} C_{i j k l} \epsilon_{k l}^{e l} \\
& \frac{\partial f^{e l}}{\partial \phi}=-p^{\prime}(\phi)\left(\epsilon_{i j}^{*, \alpha}-\epsilon_{i j}^{*, \beta}\right) C_{i j k l} \epsilon_{k l}^{e l}+\frac{1}{2} p^{\prime}(\phi) \epsilon_{i j}^{e l}\left(C_{i j k l}^{\alpha}-C_{i j k l}^{\beta}\right) \epsilon_{k l}^{e l}
\end{aligned}
$$

3.3.2. Steinbach-Apel's scheme (SAS) In SAS [11], the elastic energy is interpolated as:

$$
f^{e l}=p(\phi) f_{\alpha}^{e l}+[1-p(\phi)] f_{\beta}^{e l}
$$

with the elastic energy densities of the two phases defined as $f_{\alpha}^{e l}=\frac{1}{2} \epsilon_{i j}^{e l, \alpha} C_{i j k l}^{\alpha} \epsilon_{k l}^{e l, \alpha}$ and $f_{\beta}^{e l}=\frac{1}{2} \epsilon_{i j}^{e l, \beta} C_{i j k l}^{\beta} \epsilon_{k l}^{e l, \beta}$ respectively, where $C_{i j k l}^{\alpha}$ and $C_{i j k l}^{\beta}$ are the stiffness tensors of the two phases and elastic strains of the two phases, $\epsilon_{k l}^{e l, \alpha}$ and $\epsilon_{k l}^{e l, \beta}$, are introduced, similar to the phase compositions in the Kim et al. model (see Appendix B):

$$
\epsilon_{k l}^{e l}=p(\phi) \epsilon_{k l}^{e l, \alpha}+[1-p(\phi)] \epsilon_{k l}^{e l, \beta}
$$

The eigenstrain is interpolated as $\epsilon_{k l}^{*}=p(\phi) \epsilon_{k l}^{*, \alpha}+[1-p(\phi)] \epsilon_{k l}^{*, \beta}$. Moreover, the elastic stress is assumed to be equal in both phases at every point in the system (Reuss-Sachs' 
assumption):

$$
\begin{aligned}
& \sigma_{i j}=\sigma_{i j}^{\alpha}=\sigma_{i j}^{\beta} \\
& \Longrightarrow \epsilon_{k l}^{e l} C_{i j k l}=\epsilon_{k l}^{e l, \alpha} C_{i j k l}^{\alpha}=\epsilon_{k l}^{e l, \beta} C_{i j k l}^{\beta}
\end{aligned}
$$

From (26), (27) and (28), we get

$$
C_{i j k l}=\left[p(\phi) S_{i j k l}^{\alpha}+\{1-p(\phi)\} S_{i j k l}^{\beta}\right]^{-1}
$$

where $S_{i j k l}^{\alpha}=\left[C_{i j k l}^{\alpha}\right]^{-1}$ and $S_{i j k l}^{\beta}=\left[C_{i j k l}^{\beta}\right]^{-1}$. With this choice of interpolation scheme, equations (23) and (25) are equivalent. The derivative of the elastic energy density with respect to $\phi$ is given by (see Appendix $\mathrm{C}$ ):

$$
\frac{\partial f^{e l}}{\partial \phi}=-p^{\prime}(\phi) \sigma_{k l}\left[\left(\epsilon_{k l}^{*, \alpha}-\epsilon_{k l}^{*, \beta}\right)+\frac{1}{2}\left(\epsilon_{k l}^{e l, \alpha}-\epsilon_{k l}^{e l, \beta}\right)\right]
$$

3.3.3. Voigt-Taylor's scheme (VTS) In a VTS scheme, the total strain is assumed to be equal in the two phases at every point in the interface $\left(\epsilon_{k l}^{t o t}=\epsilon_{k l}^{t o t, \alpha}=\epsilon_{k l}^{t o t, \beta}\right)$ and the elastic stress tensor is interpolated as:

$$
\sigma_{k l}=p(\phi) \sigma_{k l}^{\alpha}+[1-p(\phi)] \sigma_{k l}^{\beta}
$$

The elastic strains of the two phases are then given by:

$$
\begin{gathered}
\epsilon_{k l}^{e l, \alpha}=\epsilon_{k l}^{t o t}-\epsilon_{k l}^{*, \alpha} \\
\epsilon_{k l}^{e l, \beta}=\epsilon_{k l}^{t o t}-\epsilon_{k l}^{*, \beta}
\end{gathered}
$$

Furthermore, it is assumed that the elastic energy is interpolated as:

$$
f^{e l}=p(\phi) f_{\alpha}^{e l}+[1-p(\phi)] f_{\beta}^{e l}
$$

Then, the derivative of the elastic energy density with respect to $\phi$ has the following form:

$$
\begin{aligned}
\frac{\partial f^{e l}}{\partial \phi} & =p^{\prime}(\phi)\left(f_{\alpha}^{e l}-f_{\beta}^{e l}\right)+p(\phi) \frac{\partial f_{\alpha}^{e l}}{\partial \phi}+[1-p(\phi)] \frac{\partial f_{\beta}^{e l}}{\partial \phi} \\
& =p^{\prime}(\phi)\left(f_{\alpha}^{e l}-f_{\beta}^{e l}\right)
\end{aligned}
$$

which is different from the formulation of Ammar et al. [15]. In their formulation, it is assumed that the stiffness tensor is interpolated as $C_{i j k l}=p(\phi) C_{i j k l}^{\alpha}+[1-p(\phi)] C_{i j k l}^{\beta}$ and elastic energy is given as $f^{e l}=\frac{1}{2} \epsilon_{i j}^{e l} C_{i j k l} \epsilon_{k l}^{e l}=p(\phi) f_{\alpha}^{e l}+[1-p(\phi)] f_{\beta}^{e l}$. However, the latter equality is not valid. Therefore, in this study, we have formulated the elastic energy as given in (33).

\subsection{Solution of elastic strain field at mechanical equilibrium}

The time scale of local displacements caused by elastic inhomogeneity in the system is much smaller than that of chemical diffusion. Therefore, the system is assumed to be at mechanical equilibrium at every time step:

$$
\frac{\partial \sigma_{i j}}{\partial r_{j}}=0
$$


The elastic stress can be written as:

$$
\begin{aligned}
\sigma_{i j}= & {\left[C_{i j k l}^{\alpha}-C_{i j k l}^{\prime}(\vec{r})\right] } \\
& {\left[\overline{\epsilon_{k l}}+\frac{1}{2}\left(\frac{\partial u_{k}(\vec{r})}{\partial r_{l}}+\frac{\partial u_{l}(\vec{r})}{\partial r_{k}}\right)-p(\phi) \epsilon_{k l}^{*, \alpha}-(1-p(\phi)) \epsilon_{k l}^{*, \beta}\right] }
\end{aligned}
$$

where $C_{i j k l}$ is rewritten as $C_{i j k l}=C_{i j k l}^{\alpha}-C_{i j k l}^{\prime}(\vec{r})$, with $C_{i j k l}^{\prime}$ being different for SAS and KHS, as given by (29) and (22) respectively. For VTS, elastic stress as given by (31) can be used directly. For all schemes, the elastic strain fields are solved at every time step using the Spectral Iterative Perturbation Method of $\mathrm{Hu}$ and Chen [20] (see Appendix D). Iterations are carried out until the displacement field converges. Heterogeneous strain is calculated from the displacement field using (5) and elastic strain field is then calculated using (2).

\subsection{Phase-field equations}

Microstructural evolution is given by the variation of the phase-field variables with time. The temporal evolution of the non-conserved phase-field variable $\phi(\vec{r}, \mathrm{t})$ is given by the Ginzburg-Landau equation:

$$
\frac{\partial \phi}{\partial t}=-L \frac{\delta F}{\delta \phi}=-L\left(\frac{\partial f^{i n t}}{\partial \phi}+\frac{\partial f^{c h}}{\partial \phi}+\frac{\partial f^{e l}}{\partial \phi}\right)
$$

where $\mathrm{L}$ is a kinetic coefficient, $\partial f^{\text {int }} / \partial \phi$ is given by (16), $\partial f^{c h} / \partial \phi$ by $(21)$, and $\partial f^{e l} / \partial \phi$ by equations (24), (30), and (35) for the three schemes respectively.

The temporal evolution of the conserved molar fraction field $\mathrm{c}(\vec{r}, \mathrm{t})$ is given by the diffusion equation:

$$
\frac{\partial c}{\partial t}=\vec{\nabla} \cdot\left(M \vec{\nabla} \frac{\delta F}{\delta c}\right)=\vec{\nabla} \cdot(M \vec{\nabla} \mu)
$$

for parabolic Gibbs free energies. $\mu$ is given by (20) and M, the chemical mobility, is assumed to be a constant and equal in the two phases in the current work.

\section{Simulation results}

The phase-field model as described in Section 3 is implemented for SAS, KHS and VTS for the model binary two-phase system in the configuration presented in figure 1(a). For the three schemes, it is validated to what extent they reproduce the findings obtained with Johnson's model (sections 2.1 and 2.2). It is first verified whether the bulk compositions, strains and stresses from the simulations agree with the analytical model. Moreover, it is analysed for the three schemes if the interpolation at the diffuse interface gives rise to excess interfacial energy or stresses, which are not present in the analytical sharp interface model. 


\subsection{Setup}

Simulations are performed for different sets of elastic properties that are chosen to analyse the origin of excess interfacial contributions. The details are listed in table 1.

For all simulations except Case III, a model system is considered. Scaled-down values of the stiffnesses of $\mathrm{Cu}_{6} \mathrm{Sn}_{5}$ and Bct-Sn phases [21,22] and chemical energies are considered so that equilibrium could be reached faster. The system size is taken as 128 $\times 4$ gridpoints, with grid size $\Delta \mathrm{x}=0.001$. The parameters $\mathrm{W}=500$ and $\kappa=0.01$ give interfacial energy $\left(\sigma_{\text {int }}\right)=0.527$ and interfacial width $\left(l_{\text {int }}\right)=0.0139$ or $13.9 \Delta \mathrm{x}$. Chemical energy is given by the parameters $A^{\alpha}=A^{\beta}=10^{5}, c_{\alpha, 0}=0.2$, and $c_{\beta, 0}=0.8$. The elastic properties used are $C_{1111}^{\alpha}=168570, C_{2222}^{\alpha}=165390, C_{1122}^{\alpha}=69110, C_{1212}^{\alpha}=$ 45981, $C_{1111}^{\beta}=82740, C_{1122}^{\beta}=57850$, and $C_{1212}^{\beta}=28180$. Eigenstrains in cases where they are non-zero are taken as $\epsilon_{11}^{*, \beta}=\epsilon_{22}^{*, \beta}=0.02$ and there are no applied strains. Other parameters are taken as $\mathrm{L}=10^{-7}, \mathrm{M}=10^{-10}$ and time step $\Delta \mathrm{t}=0.05$.

In Case III, simulations are performed for equilibrium between Bct-Sn and $\mathrm{Cu}_{6} \mathrm{Sn}_{5}$ phases from the $\mathrm{Cu}-\mathrm{Sn}$ binary system at $423 \mathrm{~K}$. The system size is taken as $128 \times$ 4 gridpoints, with grid size $\Delta \mathrm{x}=10 \mathrm{~nm}$. The parameters $\mathrm{W}=3.3 \times 10^{7} \mathrm{~J} / \mathrm{m}^{3}$ and $\kappa=1.363 \times 10^{-7} \mathrm{~J} / \mathrm{m}$ give $\sigma_{\text {int }}=0.5 \mathrm{~J} / \mathrm{m}^{2}$ and $l_{\text {int }}=20 \Delta \mathrm{x}$. Chemical energy is given by the parameters $A^{\mathrm{Cu}_{6} \mathrm{Sn}_{5}}=A^{\mathrm{Bct}}=10^{10} \mathrm{~J} / \mathrm{m}^{3}, c_{\mathrm{Sn} .0}^{\mathrm{Cu}_{6} \mathrm{Sn}_{5}}=0.455$, and $c_{\mathrm{Sn} .0}^{\mathrm{Bct}}$ $=0.999$, which give the correct equilibrium compositions at $423 \mathrm{~K}$ as computed using

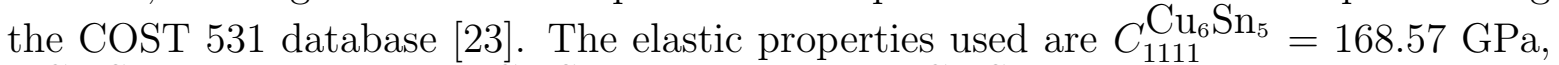
$C_{2222}^{\mathrm{Cu}_{6} \mathrm{Sn}_{5}}=165.39 \mathrm{GPa}, C_{1122} \mathrm{Cu}_{6} \mathrm{Sn}_{5}=69.11 \mathrm{GPa}, C_{1212} \mathrm{Cu}_{6} \mathrm{Sn}_{5}=45.981 \mathrm{GPa}, C_{1111}^{B c t}=82.74$ $\mathrm{GPa}, C_{1122}^{B c t}=57.85 \mathrm{GPa}$, and $C_{1212}^{B c t}=28.18 \mathrm{GPa}[21,22]$. Eigenstrains are assumed to be $\epsilon_{11}^{*}, \mathrm{Cu}_{6} \mathrm{Sn}_{5}=\epsilon_{22}^{*}, \mathrm{Cu}_{6} \mathrm{Sn}_{5}=0.5 \%$ and applied strains are zero. Other parameters are taken as $\mathrm{L}=10^{-10} \mathrm{~m}^{2} \mathrm{~s} / \mathrm{kg}, \mathrm{M}=10^{-21} \mathrm{~m}^{3} \mathrm{~s} / \mathrm{kg}$, and time step $\Delta \mathrm{t}=5 \times 10^{-6} \mathrm{~s}$.

Simulations are performed for KHS, SAS, and VTS, starting with a sharp interface and initial compositions $c_{\alpha, 0}$ and $c_{\beta, 0}$ of $\alpha$ and $\beta$ respectively. Iterations are carried out for solving the elastic field (as given in section 3.4) at every time step until the displacement field converges upto an order of $10^{-9}\left(10^{-10}\right.$ for Case III). Plane strain condition is used to calculate the compliances from the stiffnesses for 2-D [24]. All simulations are performed for $10^{6}$ time steps $\left(2.5 \times 10^{7}\right.$ time steps for Case III) to ensure that equilibrium is reached. Additional simulations are also performed to test the sensitivity of the results with change in interfacial width in the phase-field model by taking twice $(27.8 \Delta \mathrm{x})$ and thrice $(41.7 \Delta \mathrm{x})$ the initial value of interfacial width, keeping the interfacial energy fixed. This is done by choosing $\kappa$ and $\mathrm{W}$ appropriately. 


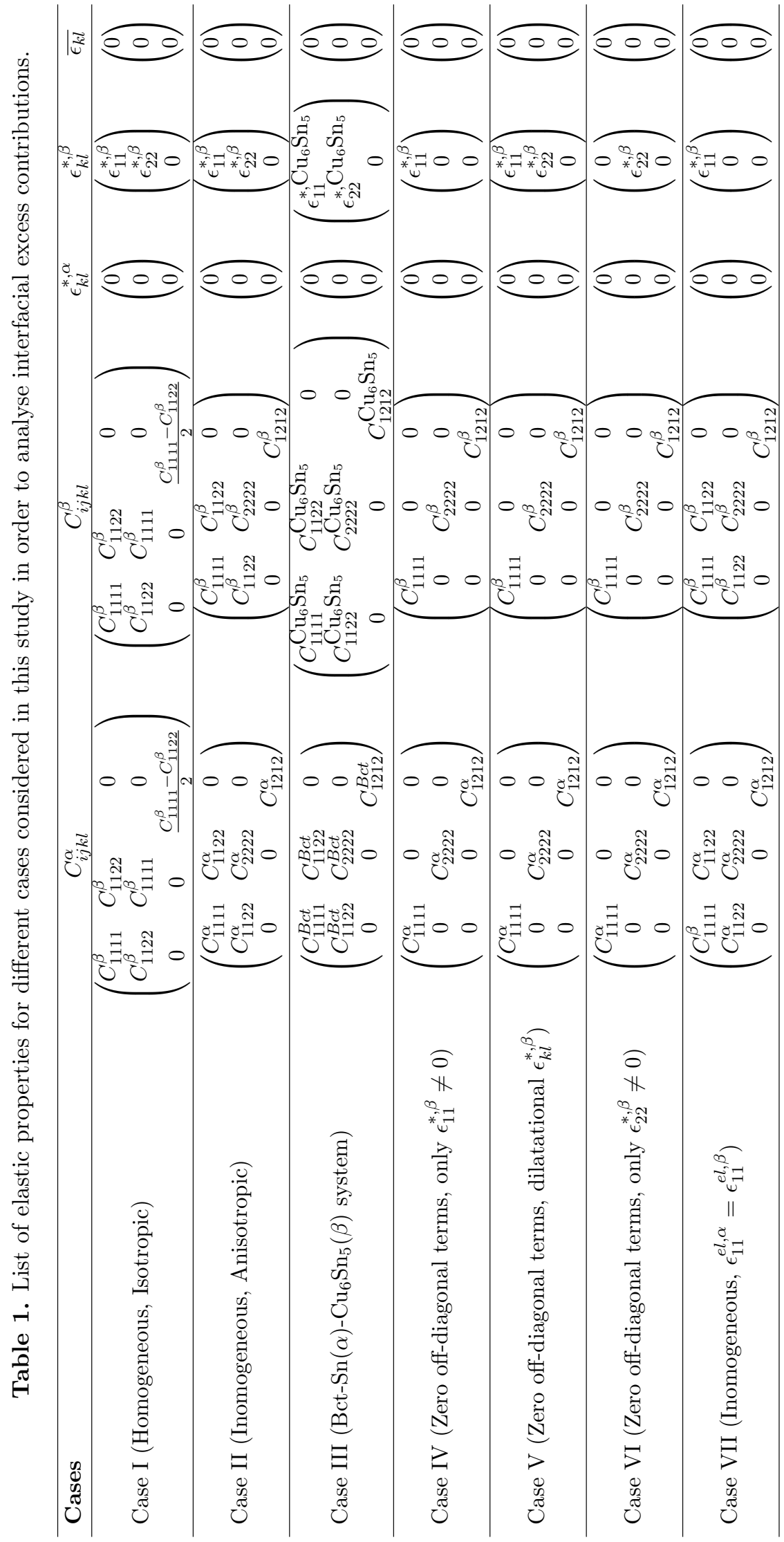




\subsection{Analysis of bulk properties}

Equilbrium compositions $c_{\alpha}^{e}$ and $c_{\beta}^{e}$ calculated using (12) and (13) and heterogeneous strains $\delta \epsilon_{11}^{\alpha}$ and $\delta \epsilon_{11}^{\beta}$ calculated using (10) and (11) from the sharp interface model compared with the values obtained from the simulations for the three schemes are listed in table 2 for all cases. The values obtained from the simulations that disagree with the analytically calculated values are indicated in italics and underlined.

Table 2. Bulk properties obtained from the simulations for the different sets of elastic properties are compared with analytical values for SAS, KHS and VTS. Compositions from simulations agree with the analytical values, but heterogeneous strains do not agree in some cases (indicated in italics and underlined) due to interfacial excess contributions.

\begin{tabular}{|c|c|c|c|c|c|c|c|c|}
\hline Bulk property & Scheme & Case I & Case II & Case III & Case IV & Case V & Case VI & Case VII \\
\hline \multirow{4}{*}{$\delta \epsilon_{11}^{\alpha}$} & KHS & -0.0169 & -0.0112 & 0.0048 & -0.0066 & -0.0066 & 0 & -0.01 \\
\hline & SAS & -0.0169 & -0.0111 & $\overline{0.0047}$ & -0.0066 & -0.0066 & 0 & -0.01 \\
\hline & VTS & $\overline{-0.0169}$ & $\overline{-0.0110}$ & 0.0048 & -0.0065 & -0.0065 & 0 & -0.01 \\
\hline & Analytical & $\overline{-0.0170}$ & $\overline{-0.0112}$ & $\overline{0.0047}$ & -0.0066 & -0.0066 & 0 & -0.01 \\
\hline \multirow{4}{*}{$\delta \epsilon_{11}^{\beta}$} & KHS & 0.0170 & 0.0112 & -0.0047 & $\underline{0.0065}$ & $\underline{0.0065}$ & 0 & 0.01 \\
\hline & SAS & 0.0170 & $\underline{0.0113}$ & -0.0047 & $\overline{0.0066}$ & 0.0066 & 0 & 0.01 \\
\hline & VTS & 0.0170 & $\underline{0.0115}$ & -0.0047 & 0.0067 & $\underline{0.0068}$ & 0 & 0.01 \\
\hline & Analytical & 0.0170 & $\overline{0.0112}$ & -0.0047 & $\overline{0.0066}$ & $\overline{0.0066}$ & 0 & 0.01 \\
\hline \multirow{4}{*}{$c_{\alpha}^{e}$} & KHS & 0.2009 & 0.2010 & 0.9981 & 0.2003 & 0.2006 & 0.2003 & 0.2006 \\
\hline & SAS & 0.2009 & 0.2010 & 0.9981 & 0.2003 & 0.2006 & 0.2003 & 0.2006 \\
\hline & VTS & 0.2009 & 0.2010 & 0.9981 & 0.2003 & 0.2006 & 0.2003 & 0.2006 \\
\hline & Analytical & 0.2009 & 0.2010 & 0.9981 & 0.2003 & 0.2006 & 0.2003 & 0.2006 \\
\hline \multirow{4}{*}{$c_{\beta}^{e}$} & KHS & 0.8009 & 0.8010 & 0.4541 & 0.8003 & 0.8006 & 0.8003 & 0.8006 \\
\hline & SAS & 0.8009 & 0.8010 & 0.4541 & 0.8003 & 0.8006 & 0.8003 & 0.8006 \\
\hline & VTS & 0.8009 & 0.8010 & 0.4541 & 0.8003 & 0.8006 & 0.8003 & 0.8006 \\
\hline & Analytical & 0.8009 & 0.8010 & 0.4541 & 0.8003 & 0.8006 & 0.8003 & 0.8006 \\
\hline
\end{tabular}

The equilibrium compositions $c_{\alpha}^{e}$ and $c_{\beta}^{e}$ are consistent with the analytical results for all schemes for all cases. Taking Case I as an example, all three schemes give identical composition profiles (figure 2(a)). This is true for other cases also. Different from our observation, Ammar et al. [15] have found that their Reuss-Sachs scheme does not give the correct equilibrium composition in the bulk phases for an elastically homogeneous system similar to Case I. This is because the $\phi$-dependence of the phase strains $\epsilon_{k l}^{e l, \alpha}$ and $\epsilon_{k l}^{e l, \beta}$ is not considered in their calculation of $\partial \epsilon_{k l}^{e l} / \partial \phi$ (see table in section 4.5 of Ammar et al. [15]).

The heterogeneous strains from all simulations are within $3 \%$ of the calculated values. The deviation from analytical values is generally larger for larger inhomogeneity in stiffnesses, absolute values of strains or change in composition. There are two main causes for deviation from the analytical values. Firstly, the analytical approach assumes 
an infinite system size, unlike the limited system size in the simulations. Therefore, there is interface movement due to the change in composition in order to maintain mass balance. This leads to a change in relative areas of the two phases, thereby causing a slight deviation in the heterogeneous strains, as per equation (11). If we take a larger system size in the simulations, this effect diminishes. This explains fully the deviation in Case I and it is consistent with the sharp interface model. Additionally, for elastically inhomogeneous systems, the deviation is also due to excess interfacial contributions introduced by the elastic energy formulation, which will be discussed in section 4.3. This is inconsistent with the sharp interface model.

The $\epsilon_{11}^{e l}$ profiles are shown for Case II in figure 2(b). Elastic strain for VTS is not shown since it cannot be calculated directly from the formulation given in section 3.3.3. The elastic energy and stress profiles for Case III are given in figures 2(c) and 2(d) respectively. For some cases, depending on the input values, there is a kink in the profiles near the diffuse interface, which is not present in the analytical model. This is due to inappropriate interpolation at the interface.

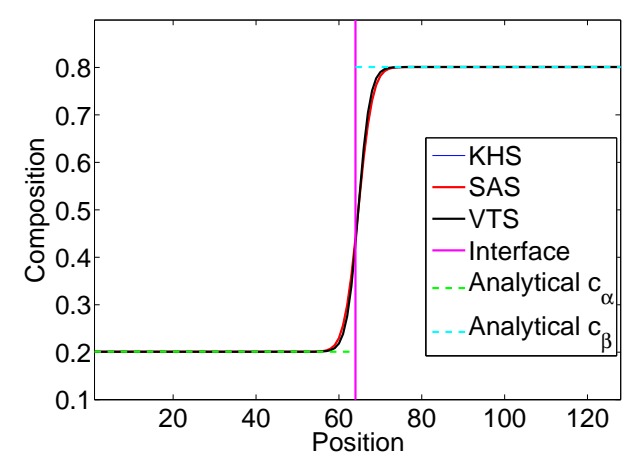

(a)

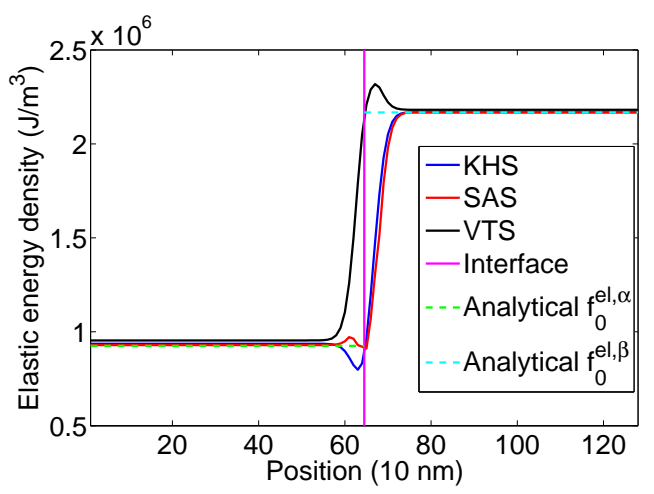

(c)

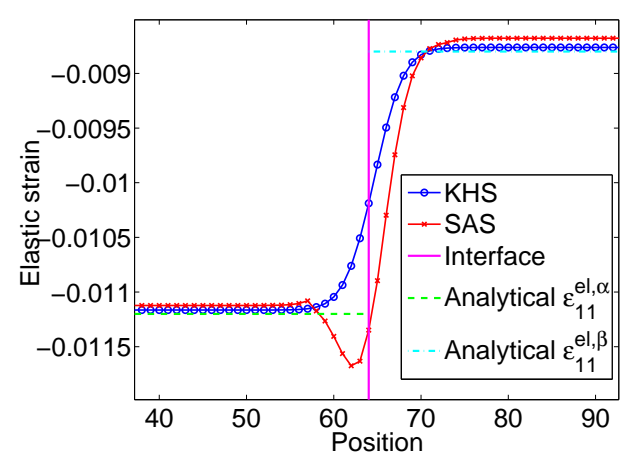

(b)

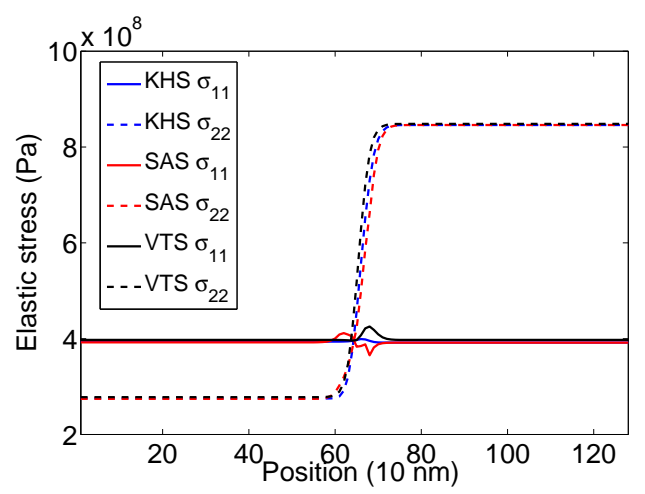

(d)

Figure 2. Comparison of the bulk properties obtained from phase-field simulations for the three schemes after $10^{6}$ time steps: (a) Composition profiles are consistent with analytical results for all cases (Case I shown here). Kinks observed in the following profiles are due to inappropriate interpolation at the interface: (b) Elastic strain $\epsilon_{11}^{e l}$ profiles of Case II. (c) Elastic energy density profiles of Case III. (d) Elastic stress profiles of Case III. 


\subsection{Analysis of interfacial excess properties}

Due to diffuse interface description, the elastic energy may give rise to an excess interfacial energy. Once the system is equilibrated, the excess elastic energy $f^{e l, x s}$, stress $\left(\sigma_{i j}^{x s}\right)$ and strain $\left(\epsilon_{i j}^{e l, x s}\right)$ can be calculated numerically from the simulations (equivalent to the Cahn-Hilliard [25] definition of interfacial energy) as:

$$
\begin{aligned}
f^{e l, x s}= & \int_{-\infty}^{+\infty} f^{e l} d l-f_{0}^{e l, \alpha} l_{\alpha}-f_{0}^{e l, \beta} l_{\beta} \\
= & \frac{l_{\text {int }}}{0.8}\left[\int_{-\infty}^{0}\left(f^{e l}-f_{0}^{e l, \alpha}\right) d \phi-\int_{0}^{+\infty}\left(f^{e l}-f_{0}^{e l, \beta}\right) d \phi\right] \\
& (\text { see Appendix E }) \\
\sigma_{i j}^{x s}= & \int_{l} \sigma_{i j} d l-\sigma_{i j}^{\alpha, 0} l_{\alpha}-\sigma_{i j}^{\beta, 0} l_{\beta} \\
\epsilon_{i j}^{e l, x s}= & \int_{l} \epsilon_{i j}^{e l} d l-\epsilon_{i j}^{e l, \alpha, 0} l_{\alpha}-\epsilon_{i j}^{e l, \beta, 0} l_{\beta}
\end{aligned}
$$

where $f^{e l}, \sigma_{i j}$ and $\epsilon_{i j}^{e l}$ are evaluated for the equilibrated system with diffuse interface, $f_{\alpha}^{e l, 0}, f_{\beta}^{e l, 0}, \sigma_{i j}^{\alpha, 0}, \sigma_{i j}^{\beta, 0}, \epsilon_{i j}^{e l, \alpha, 0}$ and $\epsilon_{i j}^{e l, \beta, 0}$ are the values obtained in the sharp interface model, and $l_{\alpha}$ and $l_{\beta}$, the lengths of the rectangular $\alpha$ and $\beta$ phases respectively, with the position of the interface taken at $\phi=0.5$. The excess stress and strain can be calculated in a similar manner, as shown by Müller and Saúl [26], under non-gliding condition of the system at mechanical equilibrium.

If the elastic energy density in the diffuse interface model is plotted with respect to the length of the system, then the two areas between the diffuse interface and sharp interface curves as marked in Figure 3 are equal in the absence of excess elastic energy. Therefore, $f^{e l}$ evolves linearly with $p(\phi)$, i.e., $f^{e l}=p(\phi) f_{0}^{e l, \alpha}+[1-p(\phi)] f_{0}^{e l, \beta}$ with the local phase quantities $f_{0}^{e l, \alpha}$ and $f_{0}^{e l, \beta}$ constant throughout the system and equal to the analytically calculated phase quantities. Excess elastic energy, if present, is proportional to the diffuse interface width, as given by (41). Our aim is to find an interpolation scheme that reproduces the findings of the sharp interface model with no excess interfacial quantities.

Excess elastic energy $\left(f^{e l, x s}\right)$, excess $\sigma_{11}\left(\sigma_{11}^{x s}\right)$, excess $\sigma_{22}\left(\sigma_{22}^{x s}\right)$, excess $\epsilon_{11}^{e l}\left(\epsilon_{11}^{x s}\right)$ and excess $\epsilon_{22}^{e l}\left(\epsilon_{22}^{x s}\right)$ are calculated according to (40)-(43) and listed in table 3 as percentages of the corresponding bulk quantities. For VTS, $\epsilon_{11}^{x s}$ and $\epsilon_{22}^{x s}$ are not listed since there is no direct relation to calculate $\epsilon_{i j}^{e l}$. The values $\sigma_{i j}^{\alpha, 0}, \sigma_{i j}^{\beta, 0}, \epsilon_{i j}^{e l, \alpha, 0}, \epsilon_{i j}^{e l, \beta, 0}, f_{0}^{e l, \alpha}$ and $f_{0}^{e l, \beta}$ are evaluated as the bulk properties in each phase obtained in the simulations.

For all the cases except Case III in all the schemes, $\sigma_{11}^{x s}$ is negligible, since for the considered geometry, $\sigma_{11}$ is equal in the two phases. However, when the magnitude of stress becomes larger, as in Case III, then a larger excess value is observed. This is reflected in the stress profiles in Figure 2(d) at the interface.

Considering Cases I, II, and III, the excess elastic energy comes from $\epsilon_{11}^{e l}, \sigma_{22}$ and $\epsilon_{22}^{e l}$. The excess energy increases with increase in interfacial width for all the schemes. For the realistic Case III with $\mathrm{Cu}_{6} \mathrm{Sn}_{5}$ and Bct-Sn phases, a large excess energy is observed 


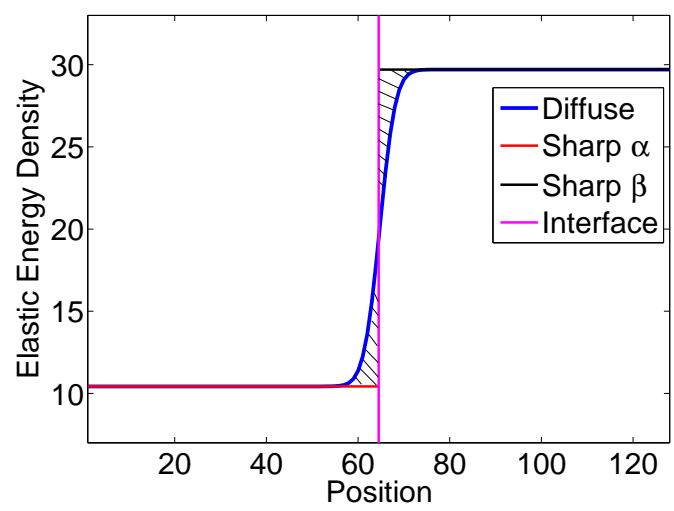

Figure 3. Variation of elastic energy with position: The two shaded areas between the diffuse interface and sharp interface curves are equal in the absence of excess energy

Table 3. Excess interfacial properties (expressed as \% of the bulk value; if bulk value $=0$, expressed as absolute value and underlined; if excess value is of the order of $10^{-4} \%$ or lesser, indicated as 0 ) obtained from simulations performed for different cases: $\sigma_{11}^{x s}$ is zero or negligible for all cases except III; Other properties are zero or negligible for SAS in Case IV, for VTS in Case VI and for KHS in Case VII.

\begin{tabular}{cccccccc}
\hline Excess properties & Case I & Case II & Case III & Case IV & Case V & Case VI & Case VII \\
\hline$f^{e l, x s} \mathrm{KHS}$ & -0.735 & -0.531 & -2.14 & -0.617 & -1.12 & -1.34 & 0 \\
$f^{e l, x s} \mathrm{SAS}$ & -0.735 & -0.333 & -2.25 & -0.0348 & -1.18 & -1.83 & 0.0092 \\
$f^{e l, x s} \mathrm{VTS}$ & 3.08 & 3.15 & 1.79 & 4.39 & 1.61 & -0.0919 & 4.42 \\
\hline$\sigma_{11}^{x s} \mathrm{KHS}$ & 0 & 0 & 0.0534 & 0 & 0 & $\underline{0}$ & 0 \\
$\sigma_{11}^{x s} \mathrm{SAS}$ & 0 & -0.0092 & 0.0367 & -0.005 & -0.0037 & $\underline{0}$ & 0 \\
$\sigma_{11}^{x s} \mathrm{VTS}$ & 0 & 0 & 0.1964 & 0 & 0 & $\underline{0}$ & 0 \\
\hline$\sigma_{22}^{x s} \mathrm{KHS}$ & -0.0939 & 1.19 & -1.30 & $\underline{0}$ & 2.27 & 2.39 & 0.0166 \\
$\sigma_{22}^{x s} \mathrm{SAS}$ & -0.0939 & 1.23 & -1.59 & $\underline{0}$ & 1.54 & 1.64 & -0.150 \\
$\sigma_{22}^{x s} \mathrm{VTS}$ & -0.0939 & 0.413 & -0.732 & $\underline{0}$ & -0.193 & -0.0919 & -0.375 \\
\hline$\epsilon_{11}^{e l s s} \mathrm{KHS}$ & 0.219 & 0.0222 & -0.962 & -0.617 & -0.662 & $\underline{0}$ & 0 \\
$\epsilon_{11}^{e l, x s} \mathrm{SAS}$ & 0.219 & 0.664 & -0.567 & -0.0349 & -0.0656 & $\underline{0}$ & 0.0094 \\
\hline$\epsilon_{2,2 l, x s}^{e l} \mathrm{KHS}$ & -0.312 & -0.342 & -0.323 & $\underline{0}$ & -0.195 & -0.092 & $\underline{0}$ \\
$\epsilon_{22}^{e l, x s} \mathrm{SAS}$ & -0.312 & -0.341 & -0.323 & $\underline{0}$ & -0.194 & -0.0919 & $\underline{0}$ \\
\hline
\end{tabular}

for all the schemes, which is inconsistent with the analytical results, in addition to inconsistent bulk elastic properties as shown in table 2. This shows that none of the schemes agrees with the analytical model for a general set of elastic properties.

In order to analyse the origin of $\sigma_{22}^{x s}$ further and, in turn, find suitable conditions such that SAS does not give excess energy, Cases IV and V are considered. We choose the stiffnesses of the two phases as containing only non-zero diagonal terms (table 1). This enables us to study the effect of $\sigma_{22}^{x s}$ independent of $\epsilon_{11}^{e l}$ and $\epsilon_{12}^{e l}$, since, in general, $\epsilon_{22}^{e l}, \epsilon_{11}^{e l}$ and $\epsilon_{12}^{e l}$ contribute to $\sigma_{22}$. Similarly, $\sigma_{11}$ is independent of $\epsilon_{22}^{e l}$ and $\epsilon_{12}^{e l}$ here. Furthermore, considering only $\epsilon_{11}^{*, \beta} \neq 0$ in Case IV, there is only one non-zero component of stress, 
$\sigma_{11}$. This ensures that SAS' condition is satisfied in the bulk phases in directions ' 1 ' and ' 2 ' $\left(\sigma_{22}^{\alpha}=\sigma_{22}^{\beta}=0\right)$ in the analytical calculations also. The elastic energy density as a function of the interpolation function $p(\phi)$ is plotted in figure $4(\mathrm{a})$ for the three schemes for three different interface widths (width1(w1) $=13.9 \Delta \mathrm{x}$, width2(w2) $=$ $27.8 \Delta \mathrm{x}$, width3(w3) $=41.7 \Delta \mathrm{x})$ for Case IV. For SAS, as expected, a linear relation independent of the interface width, is obtained, indicating no excess energy. However, this is not the case for KHS and VTS. $\sigma_{22}^{x s}$ and $\epsilon_{11}^{x s}$ are both negligible for SAS (see table 3). This proves that SAS does not give excess interfacial energy when the stiffness tensor and eigenstrain are of these specific forms. For KHS and VTS, both the bulk and interfacial excess energies depend on the interface width, as shown in figure 4(a).

In Case V, where the stiffness tensors are the same as in Case IV, but with $\epsilon_{22}^{*}$ also non-zero and equal to 0.02 , we observe that $\sigma_{22}$ is not zero. The effect of $\sigma_{22}^{x s}$ can be analysed independent of $\epsilon_{11}^{e l}$ here. There is an excess contribution from the ' 22 ' components for all the schemes (table 3 ). In SAS, $\sigma_{22}$ is assumed to be equal in both phases, which is not true in the sharp interface model (figure 1(b)), when there is nonzero eigenstrain in direction ' 2 '. Therefore, this causes the excess interfacial energy for SAS.

In Case VI, same stiffnesses as in Cases IV and V are considered, but with only $\epsilon_{22}^{*, \beta} \neq 0$ in the eigenstrain tensor. Since $\epsilon_{11}^{*, \beta}=0$, there is no heterogeneous strain generated, i.e., $\delta \epsilon_{k l}=0$. This ensures that the VTS' assumption of equal total strain (=0 here) is also valid in the bulk phases in the sharp interface model. There is no excess interfacial energy only for VTS in this case, as shown by figure 4(b).

KHS can be derived in an alternative way by introducing phase strains $\epsilon_{k l}^{e l, \alpha}$ and $\epsilon_{k l}^{e l, \beta}$ which are assumed to be equal, i.e.,

$$
\epsilon_{k l}^{e l}=\epsilon_{k l}^{e l, \alpha}=\epsilon_{k l}^{t o t}-\epsilon_{k l}^{*, \alpha}=\epsilon_{k l}^{t o t}-\epsilon_{k l}^{*, \beta}=\epsilon_{k l}^{e l, \beta}
$$

Then, considering equations (22)-(23), the elastic energy density can be written in the same form as (25) and (33). In Case VII, $C_{1111}^{\alpha}$ is taken equal to $C_{1111}^{\beta}$ and only $\epsilon_{11}^{*, \beta} \neq 0$ such that $\epsilon_{11}^{e l, \alpha}=\epsilon_{11}^{e l, \beta}$ (see table 1). This condition is derived from (10), (11) and (44). There is no excess interfacial energy only for KHS for this case as shown by figures 4(c) and $4(\mathrm{~d})$.

\section{An alternative quantitative scheme}

\subsection{Basic idea}

Based on the observations in the previous section, we propose a new scheme with strain and stress conditions at the interface which are in agreement with the analytical calculations. For the considered model 2-D system, $\sigma_{11}$ and $\epsilon_{22}^{t o t}$ are constant and equal in the two phases in the analytical model (figure 2). This condition is satisfied in the new scheme by combining SAS (equal stress) for direction ' 1 ', perpendicular to the interface, and VTS (equal total strain) for direction ' 2 ', tangential to the interface. For 


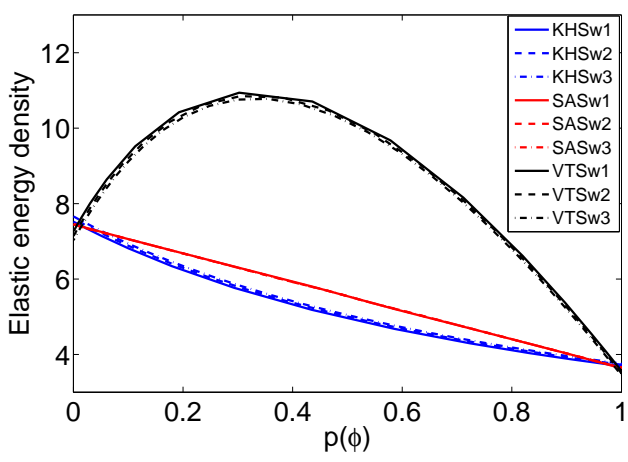

(a)

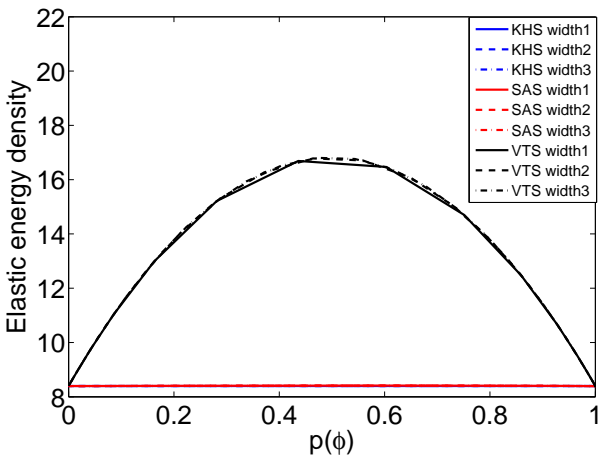

(c)

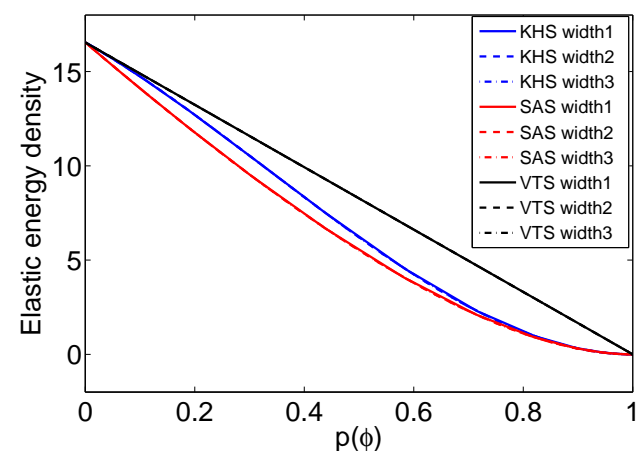

(b)

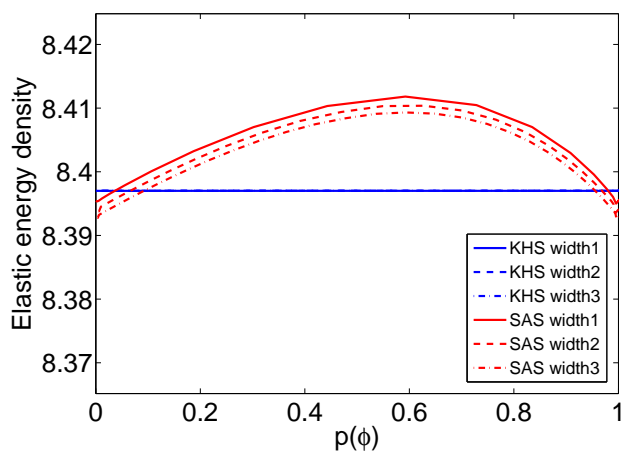

(d)

Figure 4. Elastic energy density vs $p(\phi)$ obtained from phase-field simulations after $10^{6}$ time steps for the 3 schemes and 3 different interface widths: No excess interfacial energy (linear profile) for the following: (a) only SAS for Case IV (b) only VTS for Case VI and (c) only KHS for Case VII ((d) is a zoomed-in version of (c) showing only KHS and SAS)

an arbitrary morphology in 2-D with arbitrary interface curvature, at every point on the interface, local equilibrium is maintained. The relations of equal stress and equal total strain will then hold in the directions normal to the interface and tangential to the interface respectively. Therefore, SAS and VTS can be applied in a local reference frame with coordinate axes perpendicular and tangential to the interface.

\subsection{Model formulation}

For an arbitrary morphology in 2-D, a local reference frame 1'-2' can be defined with the 1' and 2' axes perpendicular and tangential to the interface respectively as shown in figure 5 .

Let $\theta$ be the angle of rotation of the local reference frame with respect to the global 


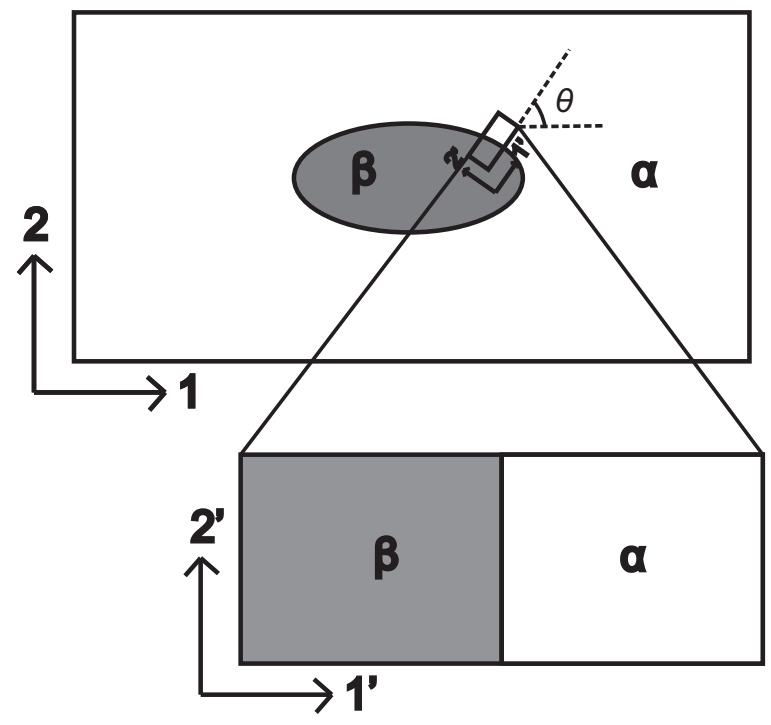

Figure 5. Representation of a local reference frame used in the new scheme, with 1' and 2' axes perpendicular and tangential to the interface respectively

reference frame 1-2. Then, according to [5],

$$
\begin{aligned}
& \cos \theta=\frac{\nabla_{x} \phi}{|\nabla \phi|} \\
& \sin \theta=\frac{\nabla_{y} \phi}{|\nabla \phi|}
\end{aligned}
$$

where $\nabla_{x} \phi$ and $\nabla_{y} \phi$ are the $\mathrm{x}$ - and $\mathrm{y}$-components of the gradient of $\phi$ respectively. The mechanical equilibrium of the system is first solved separately using SAS and VTS in the global reference frame, as described in sections 3.3.2 and 3.3.3 respectively. Let $\epsilon_{k l}^{S A S}$ and $\sigma_{k l}^{S A S}$ denote the elastic strains and stresses obtained from SAS, and $\epsilon_{k l}^{V T S}$ and $\sigma_{k l}^{V T S}$ those obtained from VTS in the global reference frame. Then, the stress, strain, stiffness and compliance tensors are transformed to the local reference frame as:

$$
\begin{aligned}
\sigma_{i j}^{S A S, \alpha^{\prime}} & =a_{i m} a_{j n} \sigma_{m n}^{S A S, \alpha} \\
\sigma_{i j}^{V T S, \alpha^{\prime}} & =a_{i m} a_{j n} \sigma_{m n}^{V T S, \alpha} \\
\epsilon_{i j}^{S A S, \alpha^{\prime}} & =a_{i m} a_{j n} \epsilon_{m n}^{S A S, \alpha} \\
\epsilon_{i j}^{V T S, \alpha^{\prime}} & =a_{i m} a_{j n} \epsilon_{m n}^{V T S, \alpha} \\
C_{i j k l}^{\alpha^{\prime}} & =a_{i m} a_{j n} a_{k o} a_{l p} C_{m n o p}^{\alpha} \\
S_{i j k l}^{\alpha^{\prime}} & =a_{i m} a_{j n} a_{k o} a_{l p} S_{m n o p}^{\alpha}
\end{aligned}
$$

where $a_{i m}$ is the rotation matrix, with elements $a_{11}=\cos \theta, a_{22}=\cos \theta, a_{12}=-\sin \theta$ and $a_{21}=\sin \theta$. Similarly, $\sigma_{i j}^{\beta}, \epsilon_{i j}^{e l, \beta}, C_{i j k l}^{\beta}$ and $S_{i j k l}^{\beta}$ are also transformed to the local reference frame.

In this local reference frame, $\sigma_{11}^{\prime}$ and $\epsilon_{22}^{\text {tot }}$ are constant and equal in the two phases and the shear components are zero as given by the analytical model (figures 5 and $2)$. According to SAS, $\sigma_{11}^{S A S, \alpha^{\prime}}=\sigma_{11}^{S A S, \beta^{\prime}}$ and according to VTS, $\epsilon_{22}^{t o t, V T S, \alpha^{\prime}}=\epsilon_{22}^{t o t, V T S, \beta^{\prime}}$. 
Using these two facts, in the new scheme, SAS in direction 1' and VTS in direction 2' are combined as follows.

In the local reference frame, the elastic stress components $\sigma_{11}^{\alpha^{\prime}}$ and $\sigma_{11}^{\beta^{\prime}}$ are both taken equal to $\sigma_{11}^{S A S^{\prime}}\left(=\sigma_{11}^{S A S, \alpha^{\prime}}=\sigma_{11}^{S A S, \beta^{\prime}}\right)$ and the total phase strains $\epsilon_{22}^{t o t, \alpha^{\prime}}$ and $\epsilon_{22}^{t o t, \beta^{\prime}}$ equal to $\epsilon_{22}^{t o t, V T S^{\prime}}\left(=\epsilon_{22}^{t o t, V T S, \alpha^{\prime}}=\epsilon_{22}^{t o t, V T S, \beta^{\prime}}\right)$. The elastic strains $\epsilon_{22}^{e l, \alpha^{\prime}}$ and $\epsilon_{22}^{e l, \beta^{\prime}}$ are then taken as $\epsilon_{22}^{V T S, \alpha^{\prime}}$ and $\epsilon_{22}^{V T S, \beta^{\prime}}$ respectively. Since shear strains are zero in the local reference frame, $\sigma_{22}^{\alpha^{\prime}}=C_{1122}^{\alpha^{\prime}} \epsilon_{11}^{e l, \alpha^{\prime}}+C_{2222}^{\alpha^{\prime}} \epsilon_{22}^{V T S, \alpha^{\prime}}$ and $\epsilon_{11}^{e l, \alpha^{\prime}}=S_{1111}^{\alpha^{\prime}} \sigma_{11}^{S A S, \alpha^{\prime}}+S_{1122}^{\alpha^{\prime}} \sigma_{22}^{\alpha^{\prime}}$. Therefore, in the new scheme, $\sigma_{22}^{\alpha^{\prime}}$ and $\epsilon_{11}^{e l, \alpha^{\prime}}$ are calculated in terms of $\sigma_{11}^{S A S, \alpha^{\prime}}$ and $\epsilon_{22}^{V T S, \alpha^{\prime}}$ as:

$$
\begin{aligned}
\sigma_{22}^{\alpha^{\prime}} & =\frac{C_{2222}^{\alpha^{\prime}} \epsilon_{22}^{V T S, \alpha^{\prime}}+C_{1122}^{\alpha^{\prime}} S_{1111}^{\alpha^{\prime}} \sigma_{11}^{S A S, \alpha^{\prime}}}{1-C_{1122}^{\alpha^{\prime}} S_{1122}^{\alpha^{\prime}}} \\
\epsilon_{11}^{e l, \alpha^{\prime}} & =\frac{S_{1111}^{\alpha^{\prime}} \sigma_{11}^{S A S, \alpha^{\prime}}+S_{1122}^{\alpha^{\prime}} C_{2222}^{\alpha^{\prime}} \epsilon_{22}^{V T S, \alpha^{\prime}}}{1-S_{1122}^{\alpha^{\prime}} C_{1122}^{\alpha^{\prime}}}
\end{aligned}
$$

Similarly, $\sigma_{22}^{\beta^{\prime}}$ and $\epsilon_{11}^{e l, \beta^{\prime}}$ can also be obtained from $\sigma_{11}^{S A S, \beta^{\prime}}$ and $\epsilon_{22}^{V T S, \beta^{\prime}}$ in the local reference frame.

The elastic energy density is interpolated as:

$$
\begin{aligned}
f^{e l}= & p(\phi) f_{\alpha}^{e l}+[1-p(\phi)] f_{\beta}^{e l} \\
= & p(\phi) \frac{1}{2}\left(\sigma_{11}^{S A S, \alpha^{\prime}} \epsilon_{11}^{e l, \alpha^{\prime}}+\sigma_{22}^{\alpha^{\prime}} \epsilon_{22}^{V T S, \alpha^{\prime}}\right) \\
& +[1-p(\phi)] \frac{1}{2}\left(\sigma_{11}^{S A S, \beta^{\prime}} \epsilon_{11}^{e l, \beta^{\prime}}+\sigma_{22}^{\beta^{\prime}} \epsilon_{22}^{V T S, \beta^{\prime}}\right)
\end{aligned}
$$

and its derivative with respect to $\phi$ is accordingly:

$$
\begin{aligned}
\frac{\partial f^{e l}}{\partial \phi}= & \frac{1}{2} p^{\prime}(\phi)\left(f_{\alpha}^{e l}-f_{\beta}^{e l}\right)+\frac{1}{2} p(\phi) \frac{\partial}{\partial \phi}\left[\sigma_{11}^{S A S, \alpha^{\prime}} \epsilon_{11}^{e l, \alpha^{\prime}}+\sigma_{22}^{\alpha^{\prime}} \epsilon_{22}^{V T S, \alpha^{\prime}}\right] \\
& +\frac{1}{2}[1-p(\phi)] \frac{\partial}{\partial \phi}\left[\sigma_{11}^{S A S, \beta^{\prime}} \epsilon_{11}^{e l, \beta^{\prime}}+\sigma_{22}^{\beta^{\prime}} \epsilon_{22}^{V T S, \beta^{\prime}}\right]
\end{aligned}
$$

where $\partial \sigma_{11}^{S A S, \alpha^{\prime}} / \partial \phi$ and $\partial \sigma_{11}^{S A S, \beta^{\prime}} / \partial \phi$ are calculated according to SAS (see Appendix F) and $\epsilon_{22}^{V T S, \alpha^{\prime}}$ and $\epsilon_{22}^{V T S, \beta^{\prime}}$ are constants with respect to $\phi$ as per VTS formulation.

Finally, the stresses and strains can be obtained in the global reference by the following transformation:

$$
\begin{aligned}
\sigma_{i j} & =a_{m i} a_{n j} \sigma_{m n}^{\prime} \\
\epsilon_{i j}^{e l} & =a_{m i} a_{n j} \epsilon_{m n}^{e l^{\prime}}
\end{aligned}
$$

For 3-D systems, a similar approach can be followed, using the appropriate rotation matrix $a_{i m}$ and expanding all the tensors with indices 1,2 and 3. A local reference frame with 1' axis normal to the interface and 2' and 3' axes in the plane tangential to the interface can be defined such that the shear strains are zero. Then, $\sigma_{33}$ and $\epsilon_{33}^{e l}$ can be treated similar to $\sigma_{22}$ and $\epsilon_{22}^{e l}$ respectively.

\subsection{Application}

Using this new scheme, simulations are performed for the general Case II (as described in table 1) with the 2-D configuration of figure 2. For this configuration, the coordinate 
transformations in the new scheme leave the tensors unchanged since the normal and the tangent to the interface are parallel to the two coordinate axes of the global reference frame; in other words, the local reference frame is same as the global one. Simulations are performed for 3 different interface widths using the same parameters as listed in section 4.1. The compositions agree with the analytical results. The heterogeneous strains obtained in the two phases using this scheme are $\delta \epsilon_{11}^{\alpha}=-0.0111$ and $\delta \epsilon_{11}^{\beta}=0.0113$. This slight deviation from the analytically calculated values $(-0.0112$ and 0.0112$)$ is, however, not due to excess interfacial energy, since the plots of $f^{e l}$ vs. $p(\phi)$ given in figure 6 show that there is no excess energy even with change in interface width for this scheme. The deviation is mainly due to the limited system size.

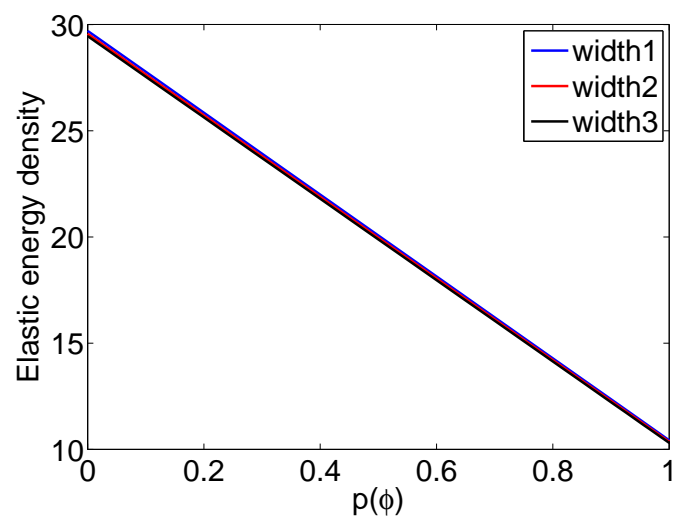

Figure 6. Elastic energy density vs. $p(\phi)$ for the general Case II using the new scheme after $10^{6}$ time steps for three different interface widths: A linear profile implies no excess interfacial energy

\section{Conclusions}

Our analysis showed that depending on the choice of interpolation scheme, interfacial excess contributions to the energy and elastic properties, varying with the diffuse interface width, may arise in phase-field models coupled with microelasticity theory. In this work, three existing interpolation schemes of bulk elastic properties in phasefield models were analysed with respect to the equilibrium bulk properties and interfacial excess contributions obtained using them.

A 2-D binary two-phase system with specific geometry is considered, for which the change in equilibrium composition in the presence of strains and the heterogeneous strain arising in the system are calculated analytically using Johnson's model. For different sets of elastic properties of the 2-D system, including a realistic $\mathrm{Cu}_{6} \mathrm{Sn}_{5}$-BctSn system, the change in equilibrium composition obtained in the phase-field simulations is consistent with the analytically calculated value for all schemes. The heterogeneous strains, however, deviate in many cases because of excess interfacial contributions and finite system size. Moreover, for some cases, including $\mathrm{Cu}_{6} \mathrm{Sn}_{5}-\mathrm{Bct}-\mathrm{Sn}$, a kink, which 
is not present in the analytical model, is observed near the interface in the profiles of elastic properties, due to inappropriate interpolation of the elastic properties in the diffuse interface.

In order to perform quantitative, realistic phase-field simulations which take into account elastic effects, a new scheme is proposed based on the analysis of the existing schemes. In the new scheme, a combination of equal total strain (VTS) in the direction parallel to the interface and equal stress (SAS) in the direction normal to the interface is used based on the interfacial conditions from the analytical model. For a general set of elastic properties, the new scheme is validated for the model 2-D system and found to be consistent with the analytical model of Johnson. In the future, this approach will be extended to simulate multi-phase multi-component systems.

\section{Acknowledgments}

The authors thank OT/07/040 'Quantitative phase-field simulation of coarsening processes in lead-free solder joints' for financial support. Simulations were performed using the VSC - Flemish Supercomputer Center, funded by the Hercules foundation and the Flemish Government - department EWI. This work was carried out within the framework of COST MP0602 Advanced Solder Materials for High Temperature Application.

\section{Appendix A. Derivative of chemical energy density with respect to $\phi$}

From equation (20),

$$
\begin{aligned}
& \frac{\partial^{2} f_{\alpha}^{c h}}{\partial^{2} c_{\alpha}}=\frac{\partial}{\partial c_{\alpha}}\left(\frac{\partial f_{\beta}^{c h}}{\partial c_{\beta}}\right) \\
&=\frac{\partial^{2} f_{\beta}^{c h}}{\partial^{2} c_{\beta}} \frac{\partial c_{\beta}}{\partial c_{\alpha}} \\
& \Rightarrow A^{\alpha}=A^{\beta} \frac{\partial c_{\beta}}{\partial c_{\alpha}} \\
& \Rightarrow \frac{\partial c_{\beta}}{\partial c_{\alpha}}=\frac{A^{\alpha}}{A^{\beta}} \\
& \frac{\partial c_{\alpha}}{\partial c_{\beta}}=\frac{A^{\beta}}{A^{\alpha}}
\end{aligned}
$$

Differentiating (19) with respect to $\phi$,

$$
\begin{aligned}
& 0=p(\phi) \frac{\partial c_{\alpha}}{\partial \phi}+[1-p(\phi)] \frac{A^{\alpha}}{A^{\beta}} \frac{\partial c_{\alpha}}{\partial \phi}+p^{\prime}(\phi)\left(c_{\alpha}-c_{\beta}\right) \\
& \Rightarrow \frac{\partial c_{\alpha}}{\partial \phi}=\frac{A^{\beta}\left(c_{\beta}-c_{\alpha}\right) p^{\prime}(\phi)}{p(\phi) A^{\beta}+[1-p(\phi)] A^{\alpha}} \\
& \frac{\partial c_{\beta}}{\partial \phi}=\frac{A^{\alpha}\left(c_{\beta}-c_{\alpha}\right) p^{\prime}(\phi)}{p(\phi) A^{\beta}+[1-p(\phi)] A^{\alpha}}[\mathrm{Using}(\mathrm{A} .4) \text { and (A.7)] }
\end{aligned}
$$


Differentiating (17) with respect to $\phi$,

$$
\frac{\partial f^{c h}}{\partial \phi}=p^{\prime}(\phi)\left(f_{\alpha}^{c h}-f_{\beta}^{c h}\right)+p(\phi) \frac{\partial f_{\alpha}^{c h}}{\partial \phi}+[1-p(\phi)] \frac{\partial f_{\beta}^{c h}}{\partial \phi}
$$

Using (18), (20), (A.7) and (A.8)],

$$
\begin{aligned}
\frac{\partial f^{c h}}{\partial \phi}= & p^{\prime}(\phi)\left(\frac{\mu_{\alpha}^{2}}{2 A^{\alpha}}-\frac{\mu_{\beta}^{2}}{2 A^{\beta}}\right) \\
& +\frac{p(\phi) \mu_{\alpha} p^{\prime}(\phi) A^{\beta}\left(c_{\beta}-c_{\alpha}\right)+[1-p(\phi)] \mu_{\beta} p^{\prime}(\phi) A^{\alpha}\left(c_{\beta}-c_{\alpha}\right)}{p(\phi) A^{\beta}+[1-p(\phi)] A^{\alpha}} \\
= & p^{\prime}(\phi)\left[\frac{\mu_{\alpha}^{2}}{2 A^{\alpha}}-\frac{\mu_{\beta}^{2}}{2 A^{\beta}}+\mu\left(c_{\beta}-c_{\alpha}\right)\right] \\
= & p^{\prime}(\phi)\left[\frac{\mu_{\alpha}^{2}}{2 A^{\alpha}}-\frac{\mu_{\beta}^{2}}{2 A^{\beta}}+\mu\left(\frac{\mu_{\beta}}{A^{\beta}}+c_{\beta, 0}-\frac{\mu_{\alpha}}{A^{\alpha}}-c_{\alpha, 0}\right)\right] \\
= & p^{\prime}(\phi)\left[f_{\beta}^{c h}-f_{\alpha}^{c h}+\mu\left(c_{\beta, 0}-c_{\alpha, 0}\right)\right]
\end{aligned}
$$

\section{Appendix B. Calculation of phase strains for SAS}

The phase strains are calculated as follows:

$$
\begin{aligned}
& \sigma_{i j}^{\alpha}=\sigma_{i j}^{\beta} \\
& \text { i.e., } \frac{\partial f_{\alpha}^{e l}}{\partial \epsilon_{i j}^{e l, \alpha}}=\frac{\partial f_{\beta}^{e l}}{\partial \epsilon_{i j}^{e l, \beta}} \\
& \Rightarrow C_{i j m n}^{\alpha} \epsilon_{m n}^{e l, \alpha}=C_{i j k l}^{\beta} \epsilon_{k l}^{e l, \beta} \\
& \Rightarrow \begin{aligned}
\Rightarrow l, \beta & =\left[C_{i j k l}^{\beta}\right]^{-1} C_{i j m n}^{\alpha} \epsilon_{m n}^{e l, \alpha} \\
& =M_{k l m n} \epsilon_{m n}^{e l, \alpha}
\end{aligned}
\end{aligned}
$$

Using (26) and (B.5),

$$
\epsilon_{k l}^{e l}=p(\phi) \epsilon_{k l}^{e l, \alpha}+[1-p(\phi)] M_{k l m n} \epsilon_{m n}^{e l, \alpha}
$$

$\epsilon_{k l}^{e l, \alpha}$ is obtained by solving (B.6). 
Appendix C. Derivative of elastic energy density with respect to $\phi$ for SAS

$$
\begin{aligned}
\frac{\partial f^{e l}}{\partial \phi} & =p^{\prime}(\phi)\left(f_{\alpha}^{e l}-f_{\beta}^{e l}\right)+p(\phi) \frac{\partial f_{\alpha}^{e l}}{\partial \phi}+[1-p(\phi)] \frac{\partial f_{\beta}^{e l}}{\partial \phi} \\
& =p^{\prime}(\phi)\left(f_{\alpha}^{e l}-f_{\beta}^{e l}\right)+p(\phi) C_{i j k l}^{\alpha} \epsilon_{i j}^{e l, \alpha} \frac{\partial \epsilon_{k l}^{e l, \alpha}}{\partial \phi} \\
& +[1-p(\phi)] C_{i j k l}^{\beta} \epsilon_{i j}^{e l, \beta} \frac{\partial \epsilon_{k l}^{e l, \beta}}{\partial \phi} \\
& =p^{\prime}(\phi)\left(f_{\alpha}^{e l}-f_{\beta}^{e l}\right)+\sigma_{k l}\left[p(\phi) \frac{\partial \epsilon_{k l}^{e l, \alpha}}{\partial \phi}+(1-p(\phi)) \frac{\partial \epsilon_{k l}^{e l, \beta}}{\partial \phi}\right] \\
& =p^{\prime}(\phi)\left(f_{\alpha}^{e l}-f_{\beta}^{e l}\right)+\sigma_{k l}\left[\frac{\partial \epsilon_{k l}^{e l}}{\partial \phi}-p^{\prime}(\phi)\left(\epsilon_{k l}^{e l, \alpha}-\epsilon_{k l}^{e l, \beta}\right)\right] \\
& =p^{\prime}(\phi)\left(\frac{1}{2} \sigma_{k l} \epsilon_{k l}^{e l, \alpha}-\frac{1}{2} \sigma_{k l} \epsilon_{k l}^{e l, \beta}\right) \\
& +\sigma_{k l}\left[-p^{\prime}(\phi)\left(\epsilon_{k l}^{*, \alpha}-\epsilon_{k l}^{*, \beta}\right)-p^{\prime}(\phi)\left(\epsilon_{k l}^{e l, \alpha}-\epsilon_{k l}^{e l, \beta}\right)\right] \\
\frac{\partial f^{e l}}{\partial \phi} & =-p^{\prime}(\phi) \sigma_{k l}\left[\left(\epsilon_{k l}^{*, \alpha}-\epsilon_{k l}^{*, \beta}\right)+\frac{1}{2}\left(\epsilon_{k l}^{e l, \alpha}-\epsilon_{k l}^{e l, \beta}\right)\right]
\end{aligned}
$$

\section{Appendix D. Hu-Chen's numerical framework}

Appendix D.1. Zeroth-order approximation

Following the method of $\mathrm{Hu}$ and Chen [20], as a zeroth-order approximation, the stiffness tensor is taken as $C_{i j k l}^{\alpha}$, a constant, ignoring $C_{i j k l}^{\prime}$, which is a function of position. Rewriting equation (36), we get

$$
\frac{1}{2} C_{i j k l}^{\alpha}\left(\frac{\partial^{2} u_{k}^{0}(\vec{r})}{\partial r_{j} \partial r_{l}}+\frac{\partial^{2} u_{l}^{0}(\vec{r})}{\partial r_{j} \partial r_{k}}\right)=C_{i j k l}^{\alpha} \frac{\partial p(\phi)}{\partial r_{j}}\left(\epsilon_{k l}^{*, \alpha}-\epsilon_{k l}^{*, \beta}\right)
$$

where $u_{k}^{0}(\vec{r})$ is the displacement field in the zeroth-order approximation. It can be solved by transformation into Fourier space:

$$
\frac{1}{2} C_{i j k l}^{\alpha}\left[i^{2} g_{j} g_{l} v_{k}^{0}(\vec{g})+i^{2} g_{j} g_{k} v_{l}^{0}(\vec{g})\right]=C_{i j k l}^{\alpha}\left(\epsilon_{k l}^{*, \alpha}-\epsilon_{k l}^{*, \beta}\right)\left[i g_{j} q(\vec{g})\right]
$$

where $v_{k}^{0}(\vec{g})$ and $q(\vec{g})$ are the Fourier transforms of $u_{k}^{0}(\vec{g})$ and $p(\phi)$ respectively. The displacement field in the real space is obtained by taking the back-Fourier transform of $v_{k}^{0}(\vec{g})$. 
Appendix D.2. Higher-order approximation

Rewriting (36) with the same left hand side as that of (D.1), we get

$$
\begin{aligned}
\frac{1}{2} C_{i j k l}^{\alpha}\left(\frac{\partial^{2} u_{k}^{n}(\vec{r})}{\partial r_{j} \partial r_{l}}+\frac{\partial^{2} u_{l}^{n}(\vec{r})}{\partial r_{j} \partial r_{k}}\right)= & \left(\epsilon_{k l}^{*, \alpha}-\epsilon_{k l}^{*, \beta}\right) C_{i j k l}^{\alpha} \frac{\partial p(\phi)}{\partial r_{j}}+\left(\overline{\epsilon_{k l}}-\epsilon_{k l}^{*, \beta}\right) \frac{\partial C_{i j k l}^{\prime}(\vec{r})}{\partial r_{j}} \\
& +\frac{1}{2} \frac{\partial}{\partial r_{j}}\left[C_{i j k l}^{\prime}(\vec{r})\left(\frac{\partial u_{k}^{n-1}(\vec{r})}{\partial r_{l}}+\frac{\partial u_{l}^{n-1}(\vec{r})}{\partial r_{k}}\right)\right] \\
& -\left(\epsilon_{k l}^{* \alpha}-\epsilon_{k l}^{*, \beta}\right) \frac{\partial}{\partial r_{j}}\left(C_{i j k l}^{\prime}(\vec{r}) p(\phi)\right)
\end{aligned}
$$

The $n$th order solution for displacement field can be obtained by substituting the $(n-1)$ th order solution on the right hand side and solving it in Fourier space. Equation (D.3) when transformed to Fourier space becomes

$$
\begin{aligned}
\frac{1}{2} C_{i j k l}^{\alpha}\left[i^{2} g_{j} g_{l} v_{k}^{n}(\vec{g})+i^{2} g_{j} g_{k} v_{l}^{n}(\vec{g})\right]= & \left(\epsilon_{k l}^{*, \alpha}-\epsilon_{k l}^{*, \beta}\right) C_{i j k l}^{\alpha}\left[i g_{j} q(\vec{g})\right]+\left(\bar{\epsilon}_{k l}-\epsilon_{k l}^{*, \beta}\right)\left[i g_{j} C_{i j k l}^{\prime}(\vec{g})\right] \\
& +\frac{1}{2} i g_{j} \operatorname{fft} 2\left[C_{i j k l}^{\prime}(\vec{r})\left\{\operatorname{ifft} 2\left(i g_{l} v_{k}^{n-1}(\vec{g})\right)+\operatorname{ifft2}\left(i g_{k} v_{l}^{n-1}(\vec{g})\right)\right\}\right] \\
& -\left(\epsilon_{k l}^{*, \alpha}-\epsilon_{k l}^{*, \beta}\right)\left[i g_{j} \operatorname{fft} 2\left(C_{i j k l}^{\prime}(\vec{r}) p(\phi)\right)\right]
\end{aligned}
$$

where fft2() and ifft2() denote forward and back-Fourier transforms respectively.

\section{Appendix E. Calculation of excess interfacial energy}

$$
\begin{aligned}
f^{e l, x s}= & \int_{-\infty}^{+\infty} f^{e l} d l-f_{0}^{e l, \alpha} l_{\alpha}-f_{0}^{e l, \beta} l_{\beta} \\
= & \int_{-\infty}^{0}\left[p(\phi) f_{\alpha}^{e l}+(1-p(\phi)) f_{\beta}^{e l}-f_{0}^{e l, \alpha}\right] d l \\
& -\int_{0}^{+\infty}\left[p(\phi) f_{\alpha}^{e l}+(1-p(\phi)) f_{\beta}^{e l}-f_{0}^{e l, \beta}\right] d l \\
= & \int_{-\infty}^{0}\left[f^{e l}-f_{0}^{e l, \alpha}\right] \frac{d l}{d \phi} d \phi-\int_{0}^{+\infty}\left[f^{e l}-f_{0}^{e l, \beta}\right] \frac{d l}{d \phi} d \phi \\
= & \frac{l_{\text {int }}}{0.8}\left[\int_{-\infty}^{0}\left(f^{e l}-f_{0}^{e l, \alpha}\right) d \phi-\int_{0}^{+\infty}\left(f^{e l}-f_{0}^{e l, \beta}\right) d \phi\right]
\end{aligned}
$$

using the definition of interfacial width $l_{\text {int }}=0.8 \mathrm{~d} l / \mathrm{d} \phi$. 


\section{Appendix F. New scheme - Calculation of elastic energy}

Expanding (57),

$$
\begin{aligned}
\frac{\partial f^{e l}}{\partial \phi}= & \frac{1}{2} p^{\prime}(\phi)\left[\sigma_{11}^{S A S, \alpha^{\prime}} \epsilon_{11}^{e l, \alpha^{\prime}}-\sigma_{11}^{S A S, \beta^{\prime}} \epsilon_{11}^{e l, \beta^{\prime}}+\sigma_{22}^{\alpha^{\prime}} \epsilon_{22}^{V T S, \alpha^{\prime}}-\sigma_{22}^{\beta^{\prime}} \epsilon_{22}^{V T S, \beta^{\prime}}\right] \\
& +\frac{1}{2} p(\phi) \frac{\partial}{\partial \phi}\left[\sigma_{11}^{S A S, \alpha^{\prime}} \epsilon_{11}^{e l, \alpha^{\prime}}+\sigma_{22}^{\alpha^{\prime}} \epsilon_{22}^{V T S, \alpha^{\prime}}\right] \\
& +\frac{1}{2}[1-p(\phi)] \frac{\partial}{\partial \phi}\left[\sigma_{11}^{S A S, \beta^{\prime}} \epsilon_{11}^{e l, \beta^{\prime}}+\sigma_{22}^{\beta^{\prime}} \epsilon_{22}^{V T S, \beta^{\prime}}\right]
\end{aligned}
$$

Since $\partial \epsilon_{22}^{e l^{\prime}} / \partial \phi=0$ as per VTS formulation, $\partial f^{e l} / \partial \phi$ is calculated using the following:

$$
\begin{aligned}
& \frac{\partial \epsilon_{11}^{e l, \alpha^{\prime}}}{\partial \phi}=\frac{S_{1111}^{\alpha^{\prime}}}{1-S_{1122}^{\alpha^{\prime}} C_{1122}^{\alpha^{\prime}}} \frac{\partial \sigma_{11}^{S A S, \alpha^{\prime}}}{\partial \phi}(\text { from }(54)) \\
& \frac{\partial \sigma_{22}^{\alpha^{\prime}}}{\partial \phi}=\frac{C_{1122}^{\alpha^{\prime}} S_{1111}^{\alpha^{\prime}}}{1-S_{1122}^{\alpha^{\prime}} C_{1122}^{\alpha^{\prime}}} \frac{\partial \sigma_{11}^{S A S, \alpha^{\prime}}}{\partial \phi}(\text { from }(53))
\end{aligned}
$$

Similar expressions are obtained for $\partial \epsilon_{11}^{e l, \beta^{\prime}} / \partial \phi$ and $\partial \sigma_{22}^{\beta^{\prime}} / \partial \phi$. Then, $\partial \sigma_{11}^{S A S, \alpha^{\prime}} / \partial \phi$ and $\partial \sigma_{11}^{S A S, \beta^{\prime}} / \partial \phi$ are calculated according to SAS as

$$
\begin{aligned}
& \frac{\partial \sigma_{11}^{S A S, \alpha^{\prime}}}{\partial \phi}= C_{1111}^{\alpha^{\prime}} \frac{\partial \epsilon_{11}^{S A S, \alpha^{\prime}}}{\partial \phi}+C_{1122}^{\alpha^{\prime}} \frac{\partial \epsilon_{22}^{S A S, \alpha^{\prime}}}{\partial \phi}+2 C_{1112}^{\alpha^{\prime}} \frac{\partial \epsilon_{12}^{S A S, \alpha^{\prime}}}{\partial \phi} \\
& \begin{aligned}
& \frac{\partial \sigma_{11}^{S A S, \beta^{\prime}}}{\partial \phi}= C_{1111}^{\beta^{\prime}} \frac{\partial \epsilon_{11}^{S A S, \beta^{\prime}}}{\partial \phi}+C_{1122}^{\beta^{\prime}} \frac{\partial \epsilon_{22}^{S A S, \beta^{\prime}}}{\partial \phi}+2 C_{1112}^{\beta^{\prime}} \frac{\partial \epsilon_{12}^{S A S, \beta^{\prime}}}{\partial \phi} \\
& \text { with } \frac{\partial \epsilon_{k l}^{S A S, \alpha^{\prime}}}{\partial \phi}= {\left[p(\phi) I+(1-p(\phi)) M_{k l m n}^{\prime}\right]^{-1} } \\
& \text { and } \frac{\partial \epsilon_{k l}^{S A S, \beta^{\prime}}}{\partial \phi}= {\left[-p^{\prime}(\phi)\left(\epsilon_{k l m n}^{*, \alpha^{\prime}}-\epsilon_{k l}^{*, \beta^{\prime}}\right)-p^{\prime}(\phi)\left(\epsilon_{k l}^{S A S, \alpha^{\prime}}-\epsilon_{k l}^{S A S, \beta^{\prime}}\right)\right] } \\
& \partial \phi
\end{aligned}
\end{aligned}
$$

where $M_{k l m n}^{\prime}=\left[C_{i j k l}^{\beta^{\prime}}\right]^{-1} C_{i j m n}^{\alpha^{\prime}}$.

\section{References}

[1] Cahn J W and Larché F 1984 A simple model for coherent equilibrium Acta Metall. 32(11) $1915-23$

[2] Pfeifer M J and Voorhees P W 1991 A graphical method for constructing coherent phase diagrams Acta Metall. Mater. 39(8) 2001-12

[3] Johnson W C 1987 Precipitate shape evolution under applied stress - thermodynamics and kinetics Metall. Trans. A 18A 233-47

[4] Chen L-Q 2002 Phase-field models for microstructure evolution Annu. Rev. Mater. Res. 32 113-40

[5] Moelans N, Blanpain B, and Wollants P 2008 An introduction to phase-field modeling of microstructure evolution Calphad 32 268-94

[6] Steinbach I 2009 Phase-field models in materials science Modelling Simul. Mater. Sci. Eng. 17 073001

[7] Khachaturyan A G 1983 Theory of structural transformations in solids (New York: Wiley)

[8] Seol D J, Hu S Y, Oh K H, and Chen L-Q 2004 Effect of substrate constraint on spinodal decomposition in an elastically inhomogeneous thin film Met. Mater. Int. 10(5) 429-34 
[9] Greenwood M, Hoyt J J, and Provatas N 2009 Competition between surface energy and elastic anisotropies in the growth of coherent solid-state dendrites Acta Mater. 57 2613-23

[10] Tonks M, Millett P, Cai W, and Wolf D 2010 Analysis of the elastic strain energy driving force for grain boundary migration using phase field simulation Scripta Mater. 63 1049-52

[11] Steinbach I and Apel M 2006 Multi phase field model for solid state transformation with elastic strain Physica D 217 153-60

[12] Steinbach I and Apel M 2007 The influence of lattice strain on pearlite formation in Fe-C Acta Mater. 55 4817-22

[13] Guo W, Steinbach I, Somsen C, and Eggeler G 2011 On the effect of superimposed external stresses on the nucleation and growth of $\mathrm{Ni}_{4} \mathrm{Ti}_{3}$ particles: A parametric phase field study Acta Mater. $593287-96$

[14] Kim D-U, Kim S G, Kim W T, Cho J, Han H-N, and Cha P-R 2010 Effect of micro-elasticity on grain growth and texture evolution: a phase field study Mater. Sci. Forum 654-656 1590-3

[15] Ammar K, Appolaire B, Cailletaud G, and Forest S 2009 Combining phase field approach and homogenization methods for modelling phase transformation in elastoplastic media Eur. J. Comput. Mech. 18(5-6) 485-523

[16] Hill R 1963 Elastic properties of reinforced solids: Some theoretical principles J. Mech. Phys. Solids $11357-72$

[17] Durga A and Moelans N 2012 Handbook of High-Temperature Lead-Free Solders, Volume 3: Group Project Reports, ed Kroupa A (COST) chapter Effect of elastic stresses on the growth of IMC precipitates - phase-field method coupled with micro-elasticity theory

[18] Kim S G, Kim W T, and Suzuki T 1999 Phase-field model for binary alloys Phys. Rev. E 60(6) 7186-96

[19] Wang S L, Sekerka R F, Wheeler A A, Murray B T, Coriell S R, Braun R J, and McFadden G B 1993 Thermodynamically-consistent phase-field models for solidification Physica D 69 189-200

[20] Hu S Y and Chen L-Q 2001 A phase-field model for evolving microstructures with strong elastic inhomogeneity Acta Mater. 49 1879-90

[21] Chen J and Lai Y-S 2009 Towards elastic anisotropy and strain-induced void formation in Cu-Sn crystalline phases Microelectron. Reliab. 49 264-8

[22] Ghosh G 2009 First-principles calculation of phase stability and cohesive properties of Ni-Sn intermetallics Metall. Mater. Trans. A 40A 4-23

[23] COST Action 531-Atlas of Phase Diagrams for Lead-free Solders, vol 1 ed Dinsdale A T, Watson A, Kroupa A, Vrestal J, Zemanova A, J. Vizdal (Brno, Czech Republic)

[24] Little R W 1973 Elasticity (New Jersey: Prentice-Hall, Inc.)

[25] Cahn J W and Hilliard J E 1958 Free energy of a nonuniform system. I. Interfacial energy J. Chem. Phys. 28(2) 258-67

[26] Müller P and Saúl A 2004 Elastic effects on surface physics Surf. Sci. Rep. 54 157-258 